\title{
Integral field spectroscopy of nitrogen overabundant blue compact dwarf galaxies
}

\author{
E. Pérez-Montero ${ }^{1}$, J. M. Vílchez ${ }^{1}$, B. Cedrés ${ }^{1}$, G. F. Hägele ${ }^{2,3, \star}$, M. Mollá ${ }^{4}$, \\ C. Kehrig ${ }^{5}$, A. I. Díaz ${ }^{2}$, R. García-Benito ${ }^{6}$, and D. Martín-Gordón ${ }^{1}$ \\ ${ }^{1}$ Instituto de Astrofísica de Andalucía - CSIC. Apdo. 3004, 18008 Granada, Spain \\ e-mail: [epm; jvm;bce; dmg] @iaa.es \\ 2 Departamento de Física Teórica, C-XI, Universidad Autónoma de Madrid, 28049 Cantoblanco, Madrid, Spain \\ e-mail: [guille.hagele; angeles.diaz]@uam.es \\ 3 Facultad de Cs. Astronómicas y Geofísicas., Universidad Nacional de La Plata, Paseo del Bosque s/n, 1900 La Plata, Argentina \\ ${ }^{4}$ Departamento de Investigación Básica, CIEMAT, Avda. Complutense 22, 28040 Madrid, Spain \\ e-mail: mercedes.molla@ciemat.es \\ 5 Leibniz-Institut für Astrophysik Postdam, innoFSPEC Postdam, An der Sternwarte 16, 14482 Postdam, Germany \\ e-mail: ckehrig@aip.de \\ ${ }^{6}$ Kavli Institute of Astronomy and Astrophysics, Peking University, 100871 Beijing, PR China \\ e-mail: luwen@pku.edu.cn
}

Received 25 January 2011 / Accepted 14 May 2011

\section{ABSTRACT}

\begin{abstract}
Context. The oxygen and nitrogen-to-oxygen abundances relation is characterized by a plateau around $\log (\mathrm{N} / \mathrm{O})=-1.6$ at metallicities lower than $12+\log (\mathrm{O} / \mathrm{H}) \sim 8.25$. However, there is a subset of blue compact dwarf galaxies $(\mathrm{BCDs})$ with unexpectedly high $\mathrm{N} / \mathrm{O}$ values.

Aims. We want to study the spatial distribution of the physical properties and of oxygen and nitrogen abundances in three BCDs (HS 0128+2832, HS 0837+4717, and Mrk 930) with a reported excess of N/O in order to investigate the nature of this excess and, particularly, its association with Wolf-Rayet (WR) stars.

Methods. We observed these BCDs by using PMAS integral field spectroscopy in the optical spectral range (3700-6900 A) and mapping the brightest emission lines, hence their interstellar medium (ISM) physical-chemical properties (reddening, excitation, electron density and temperature, and $\mathrm{O}$ and $\mathrm{N}$ chemical abundances), using both the direct method and appropriate strong-line methods. We made a statistical analysis of the resulting distributions and compared them with the integrated properties of the galaxies. Results. Outer parts of the three galaxies are placed in the "AGN-zone" of the $[\mathrm{NII}] / \mathrm{H} \alpha$ vs. $[\mathrm{OIII}] / \mathrm{H} \beta$ diagnostic diagram most likely due to a high N/O combined with the excitation structure in these regions. From the statistical analysis, it is assumed that a certain property can be considered as spatially homogeneous (or uniform) if a normal Gaussian function fits its distribution in several regions of the galaxy. Moreover, a disagreement between the integrated properties and the mean values of the distribution usually appears when a Gaussian does not fit the corresponding distribution. We find that 1) for HS $0128+2832$, only the N/O ratio as derived from the direct method is uniform; 2) for HS $0837+4717$, all properties are uniform; and 3) for Mrk 930, the uniformity is found for all parameters, except for electron density and reddening. The rotation curve, the $\mathrm{H} \alpha$ map, and UV images reveal a perturbed morphology and possible interacting processes in Mrk 930. Finally, if the N/O is constant on spatial scales of several kpc, as our analysis indicates, we conclude that there are not enough WR stars estimated from spectroscopy to pollute the ISM and to produce the observed N/O excess in these objects.
\end{abstract}

Key words. galaxies: evolution - galaxies: abundances - galaxies: starburst - galaxies: kinematics and dynamics - galaxies: stellar content

\section{Introduction}

The understanding of the formation and evolution of starburst galaxies has so far been based mainly on the study of different integrated properties of their star-forming knots, which depend on their content of stars, gas, and dust. In the optical spectrum of these knots, the blue light emitted by the massive young star clusters and the emission lines coming from the ionized gas stand out. These lines provide information about the physical properties and the chemical abundances of the gas and the stars. Between the different types of starburst galaxies, blue compact dwarf galaxies (BCDs), also known as HII galaxies, were

\footnotetext{
* CONICET, Argentina.
}

characterized very accurately by analyzing their optical spectra in a wide sample of these objects (e.g., Terlevich et al. 1981; Kunth \& Östlin 2000). Since the metallicity distribution of these objects peaks at a low value (about $1 / 5 Z_{\odot}$ ), the cooling rate in the ionized gas is much less efficient than in metal-rich giant HII regions in galaxies disks, and consequently their electron temperatures are much higher. This makes determination of chemical abundances using the so-called direct method (i.e. using collisionally excited lines and electron temperatures) much more accurate than in other metallicity regimes (e.g., Pérez-Montero \& Díaz 2003; Hägele et al. 2006). This feature of BCDs, together with their compact aspect and gas richness, make them ideal scenarios for studying the star formation processes in the local universe and in low-metallicity environments resembling 
those in a younger Universe. Particularly, it is interesting to look into the ionic abundance ratios between species with an assumed different stellar origin to probe the chemical evolution of these objects.

This is the case of the nitrogen-to-oxygen ratio $(\mathrm{N} / \mathrm{O})$, since their stellar yields are quite different and mostly proceed from low- and intermediate-mass and massive stars, respectively. Therefore, it gives important clues to the study of the star formation rate and history of star-forming galaxies (Mollá et al. 2006). This ratio has already been well studied for decades. Edmunds \& Pagel (1978) and Alloin et al. (1979) concluded that the constant value of $\mathrm{N} / \mathrm{O}[\log (\mathrm{N} / \mathrm{O}) \sim-1.6]$ in the low-metallicity regime (i.e. for $12+\log (\mathrm{O} / \mathrm{H})<8$ ) is consistent with a primary origin of the nitrogen, while the correlation between $\mathrm{N} / \mathrm{O}$ and $\mathrm{O} / \mathrm{H}$ at higher oxygen abundances corresponds to a secondary production of $\mathrm{N}$. These findings were later confirmed by McCall et al. (1985) and Vila-Costas \& Edmunds (1993), among others.

Traditionally, both the plateau and the dispersion found in the $\mathrm{N} / \mathrm{O}$ vs. $\mathrm{O} / \mathrm{H}$ plot at the low-metallicity regime were explained in terms of the delay between the ejections of $\mathrm{O}$ and $\mathrm{N}$, which appears if $\mathrm{O}$ is produced by massive stars and $\mathrm{N}$ by low- and intermediate-mass stars. In that case, the different star formation histories included in chemical evolution models may change the exact value of $\mathrm{O} / \mathrm{H}$ for which the secondary $\mathrm{N}$ would appear and, therefore, the observed dispersion is easily explained, as Henry et al. (2000), Prantzos (2003) and Mollá et al. (2006) found. At the same time the plateau appears as a consequence of a very low and continous star formation rate and of metallicity-dependent stellar yields, which, as expected, give a high proportion of primary $\mathrm{N}$ when $\mathrm{O} / \mathrm{H}$ is low, and a higher secondary $\mathrm{N}$ production when $\mathrm{O} / \mathrm{H}$ increases.

Besides this, the relative production of $\mathrm{N}$ is sensitive to the assumed theoretical stellar yields. Thus, Renzini \& Voli (1981) predicted a certain amount of primary $\mathrm{N}$ produced by the lowand intermediate-mass stars. Other works also give a proportion of primary $\mathrm{N}$ for this stellar mass range: Van der Höek \& Groenewegen (1997) and, more recently, Gavilán et al. (2006). These stellar yields, computed for a wide range of metallicities and masses and by simplified synthesis stellar models were also corroborated by precise calculations from the stellar evolution field (Marigo 2001; Ventura et al. 2002; Dray 2003). Additionally, Meynet \& Maeder (2002) and Chiappini et al. (2006) predict an enhancement of the N production in the lowmetallicity regime assuming rapid rotation of massive and intermediate mass stars and fitting the observations coming from the halo stars of our Galaxy.

In any case, by assuming a recursive star formation history with several bursts, it is also possible to find some extra enhancements of the $\mathrm{N} / \mathrm{O}$ ratio in the starburst phases: Oxygen increases when massive stars die, simultaneously decreasing $\mathrm{N} / \mathrm{O}$, and then, when intermediate mass stars die, $\mathrm{N}$ increases again. Some peaks should thus appear in the O/H vs. N/O diagram (Garnett 1990; Pilyugin 1992). Several authors appealed to hydro-dynamical effects, such as outflows of enriched gas by super-massive galactic winds (van Zee et al. 1998) or inflows of metal-poor gas (Köppen \& Hensler 2005), which makes the metallicity of the galaxy lower, keeping the N/O ratio at a high value, to explain the observations.

In this context, there is a subsample of BCDs that present high values of $\mathrm{N}$ in comparison to the expected value for their metal content, even taking the observational dispersion into account (Pérez-Montero \& Contini 2009). Two examples are Mrk 996 (Thuan et al. 1996, $12+\log (\mathrm{O} / \mathrm{H})=7.96, \log (\mathrm{N} / \mathrm{O})=-0.80)$ and $\mathrm{NGC} 5253$
(Walsh \& Roy 1987, 1989; Kobulnicky et al. 1997, $12+\log (\mathrm{O} / \mathrm{H})=8.12, \log (\mathrm{N} / \mathrm{O})=-0.83$ in the region HII-1). More recently, "Green pea" galaxies, which resemble BCDs in many aspects, were found to also be depicted by low metallicities and high N/O (Amorín et al. 2010). In a number of these, metal-poor BCDs with an enhanced N/O has been detected broad prominent emissions at $\sim 4650 \AA$ and $\sim 5808 \AA$. These broad features are emitted by Wolf-Rayet (WR) stars, which are characterized by the ejection of material via strong stellar winds that pollute the interstellar medium (ISM) with the products of the $\mathrm{H}$ and He-burning in the outer layers of these stars. This suggests that WR stars could also be the responsible for the high $\mathrm{N}$ abundances in these galaxies.

Among BCDs showing high N/O and where WR stars were detected, there are UM420, UM448, the merger compact group Mrk 1089 (all these in Guseva et al. 2000), HS 0837+4717 (Pustilnik et al. 2004), and NGC 5253 (López-Sánchez et al. 2007; Monreal-Ibero et al. 2010). In the study of galaxies with WR signatures from the Sloan Digital Sky Survey (SDSS) carried out by Brinchmann et al. (2008), it is also found that WR galaxies show an elevated N/O relative to non-WR galaxies. Additionally, an N/O excess is reported in other six BCDs with $\log (\mathrm{N} / \mathrm{O})>-1.3$ and a prominent WR blue bump in their optical integrated spectra (Hägele et al. 2006, 2008; Pérez-Montero et al. 2010). On the other hand, the high N/O found in metalpoor halo stars indicates that another mechanism polluting the ISM with N, other than WR star winds, might exist.

Nevertheless, most of the contributions that establish the relation between the local $\mathrm{N}$ pollution and the presence of WR stars are based on long-slit integrated observations, and therefore, almost no information about the relative spatial position of massive stars and possible chemical inhomogeneities exist in this sample of BCDs. One of the few examples is described in Kehrig et al. (2008) who found an excess of N/O simultaneously with the detection of WR stellar population across the BCD IIZw70 by means of integral field spectroscopy (IFS). These are also the cases of NGC5253 (Monreal-Ibero et al. 2010) and IC10 (LópezSánchez et al. 2011). IFS constitutes a powerful tool for studying the spatial distribution of the different physical properties and chemical abundances across the objects in detail. Besides this, an appropriate statistical analysis of these distributions helps to distinguish between the spatial uniformity of some properties and to detect local inhomogeneity or pollution of chemical elements in the ISM of galaxies, and possibly to link them with the positions and properties of the star-forming knots.

In this work, we use IFS to analyze three BCDs (HS 0128+2832, HS 0837+4717, and Mrk 930) selected because of their overabundance of $\mathrm{N}$ reported in the literature. In HS $0837+4717$ and Mrk 930, the presence of WR stars has been confirmed by long-slit spectroscopy. IFS leads to a $2 \mathrm{D}$ spatial characterization of both the stellar population and the ISM physical-chemical properties that allows us to check whether the $\mathrm{N} / \mathrm{O}$ excess is spatially correlated with the WR stars or if it is due to a more global process affecting the whole galaxy.

The paper is organized as follows. In Sect. 2, we describe our sample of objects as well as the observations and data reduction. In Sects. 3 and 4, we present and discuss our results. Finally, Sect. 5 summarizes the main conclusions derived from this work. 
Table 1. List of the observed objects, with some additional basic information taken from Izotov \& Thuan (2004; HS 0128+2832), Pustilnik et al. (2004; HS 0837+4717) and Izotov \& Thuan (1998; Mrk 930).

\begin{tabular}{lcccccccc}
\hline \hline Object ID & Other names & RA $(2000)$ & $\delta(2000)$ & $D(\mathrm{Mpc})$ & Redshift & $\log \mathrm{F}(\mathrm{H} \alpha)$ & $12+\log (\mathrm{O} / \mathrm{H})$ & $\log (\mathrm{N} / \mathrm{O})$ \\
\hline HS 0128+2832 & & $01^{\mathrm{h}} 31^{\mathrm{m}} 21.3^{\mathrm{s}}$ & $+28^{\mathrm{d}} 48^{\mathrm{m}} 12^{\mathrm{s}}$ & 63.1 & 0.016130 & -13.23 & $8.12 \pm 0.05$ & $-1.23 \pm 0.14$ \\
HS 0837+4717 & \multirow{2}{*}{ SHOC 220 } & $08^{\mathrm{h}} 40^{\mathrm{m}} 29.9^{\mathrm{s}}$ & $+47^{\mathrm{d}} 07^{\mathrm{m}} 10^{\mathrm{s}}$ & 166.0 & 0.041950 & -13.06 & $7.62 \pm 0.06$ & $-0.75 \pm 0.20$ \\
Mrk 930 & & $23^{\mathrm{h}} 31^{\mathrm{m}} 58.3^{\mathrm{s}}$ & $+28 \mathrm{~d} 56 \mathrm{~m} 50 \mathrm{~s}$ & 83.2 & 0.018296 & -12.71 & $8.07 \pm 0.03$ & $-1.42 \pm 0.10$ \\
\hline
\end{tabular}

\section{Data}

\subsection{The sample}

Three BCDs with different degrees of $\mathrm{N}$ overabundance, which were derived using integrated long-slit spectroscopy available in the literature and visible from the northern hemisphere at the epoch of observations, were selected. The main properties of these objects as taken from the literature are listed in Table 1, including names, position, adopted distance, redshift and $\mathrm{H} \alpha$ flux in the slit without aperture correction. The available data comprise the required emission lines to derive at least one electron temperature allowing the direct and precise determination of the $\mathrm{O}$ and $\mathrm{N}$ chemical abundances. These were recalculated using the methodology described in the Sect. 3.6, and they are also listed in Table 1. In Fig. 1, we show the derived $\mathrm{O}$ abundance and N/O for these three objects compared to the values from the star-forming galaxies of the SDSS (Amorín et al. 2010). As we see, all of them present values of N/O higher than the average $\mathrm{N} / \mathrm{O}$ for their same metallicity regime.

HS $0128+2832$ is a compact galaxy studied by Izotov $\&$ Thuan (2004), who derived a low $\mathrm{O}$ abundance $[12+$ $\log (\mathrm{O} / \mathrm{H})=8.12]$ combined with an elevated abundance of $\mathrm{N}$ $[\log (\mathrm{N} / \mathrm{O})=-1.23]$. No trace of WR stars was reported in the integrated spectrum of this object. HS $0837+4717$ is apparently another compact galaxy that has been extensively studied by Pustilnik et al. (2004), who found very low O abundance [12 $+\log (\mathrm{O} / \mathrm{H})=7.65]$ and a very high value of the relative abundance of $\mathrm{N}[\log (\mathrm{N} / \mathrm{O})=-0.75]$. These authors detected the WR blue bump, which was used to estimate approximately $1000 \mathrm{WR}$ stars in the starburst. Although the stellar origin of the HeII line shown by Pustilnik is not clear, this galaxy also appears in the catalog of WR objects in the SDSS (WR101) as a class 2 object (i.e. convincing WR feature but not obvious after continuum subtraction). Our third object, Mrk 930, presents a quite disturbed morphology, characterized by an elongated shape, whose main burst of star formation is in the south, but other several fainter knots are also visible in the northern part. Figure 2 shows an ACS-HST $^{1}$ image taken with the F140LP filter centered on the ultraviolet at a wavelength $\sim 1400 \AA$, where the presence of these super star clusters is remarkable along the galaxy in a complex structure. Guseva et al. (2000) analyzed both the blue and red WR bumps detected by Izotov \& Thuan (1998) for this galaxy, and they estimated around 2000 WR stars, although this is very uncertain due to the noise of the spectrum. A very noisy hint of this WR feature is also pointed out by Adamo et al. (2011) using long-slit spectroscopy of the brightest knot of this galaxy. The chemical analysis carried out by Izotov \& Thuan (1998) for this galaxy revealed a low metallicity $[12+\log (\mathrm{O} / \mathrm{H})=8.07]$ and a slightly enhanced value of the N/O $[\log (\mathrm{N} / \mathrm{O})=-1.42]$.

\footnotetext{
1 This image has been taken from the Multimission Archive at STScI (MAST) in the webpage http://archive.stsci.edu/
}

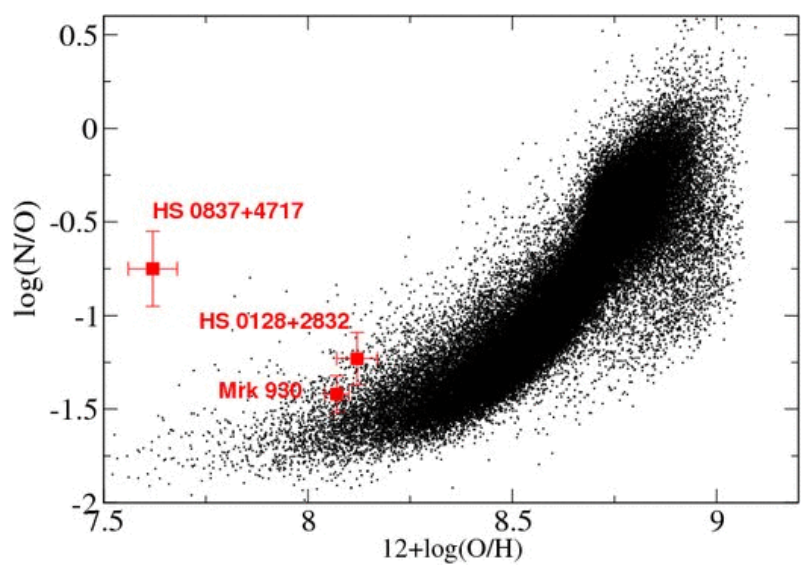

Fig. 1. Relation between $\mathrm{O}$ abundance and N/O ratio as derived from emission-line spectra of star-forming objects of the SDSS. The red squares represent the objects of the sample described in the text, with their values recalculated using the emission lines reported in the literature.

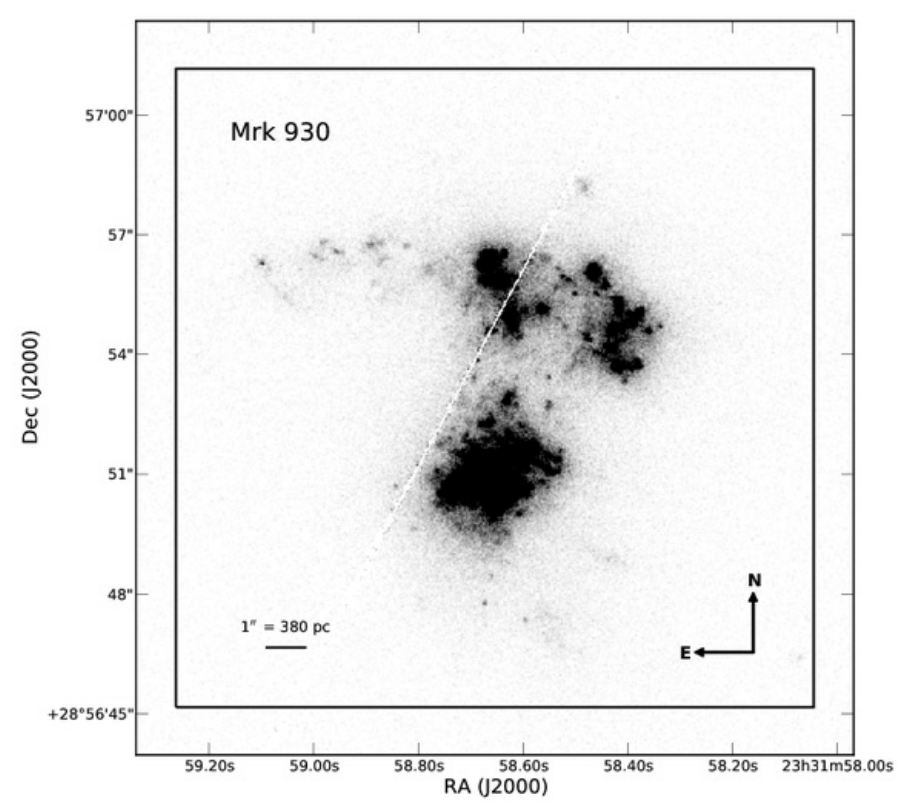

Fig. 2. HST-ACS image of Mrk 930 taken with the $F 140-L P$ filter. The square shows the field of view of the PMAS instrument encompassing the whole galaxy. The figure also shows the complex structure of the ionizing stellar clusters in this galaxy. North points up and east to the left.

The study of these three galaxies by means of IFS constitutes an ideal test to find out to what extent the relative enrichment of $\mathrm{N}$ found in some BCDs is related to the presence of WR stars in their knots of star formation. 
Table 2. Log of observations.

\begin{tabular}{lcc}
\hline \hline Object & $\begin{array}{c}B \text { exptime } \\
(\mathrm{s})\end{array}$ & $\begin{array}{c}R \text { exptime } \\
(\mathrm{s})\end{array}$ \\
\hline HS 0128+2832 & $2 \times 1200+3 \times 900$ & $2 \times 1200+3 \times 900$ \\
HS 0837+4717 & $2 \times 1200+1 \times 900$ & $2 \times 1200+1 \times 900$ \\
Mrk 930 & $2 \times 1200$ & $2 \times 1200$ \\
\hline
\end{tabular}

\subsection{Observations}

Observations were carried out using the integral field unit (IFU) Postdam Multi-Aperture Spectrophotometer (PMAS), developed at the Astrophysikalisches Institut Potsdam (Roth et al. 2005). PMAS is attached to the $3.5 \mathrm{~m}$ Telescope in the Calar Alto Observatory (Almería, Spain). The data were acquired on 2008 October 29, under good conditions of transparency and with a typical seeing close to $2^{\prime \prime}$. To avoid major differential atmospheric refraction effects, all expositions were taken at an air mass lower than 1.2.

One single pointing was taken for each galaxy, covering in all cases the most intense burst of star formation and its surroundings.

The log of observations is given in Table 2. The V600 grating, with a dispersion of $1.6 \AA /$ pixel in the $2 \times 2$ binning mode to reduce the reading time and readout noise, was used in two spectral ranges, covering both the blue (3700-5200 $\AA$ ) and red (5350-6850 $\AA$ ) sides. The PMAS spectrograph is equipped with 256 fibers coupled to a $16 \times 16$ lens array. Each fiber has a spatial sampling of $1^{\prime \prime} \times 1^{\prime \prime}$ on the sky, resulting in a field of view of $16^{\prime \prime} \times 16^{\prime \prime}$. This field is shown in relation to the size of Mrk 930 in Fig. 2. At the reported distances of the three objects (see Table 1), each spaxel size of 1" corresponds to $330 \mathrm{pc}$ in HS $0128+2832,840$ pc in HS 0837+4717, and 380 pc in Mrk 930. Calibration images were taken following the science exposures and consisted of emission line lamp spectra $(\mathrm{HgNe})$ and spectra of a continuum lamp needed to locate the 256 individual spectra on the CCD. Observations of the spectrophotometric standard stars $\mathrm{BD}+28^{\circ} 4211$ and $\mathrm{Hz} 44$ were taken during the observing night for flux calibration.

\subsection{Data reduction}

We reduced the data using the software R3D (Sánchez 2006). Different exposures taken at the same pointing were combined using IRAF ${ }^{2}$ tasks. The expected locations of the spectra were traced on a continuum-lamp images taken before each target exposure. After bias subtraction, we extracted the target spectra by adding the signal from the five pixels around the central traced pixel (which is the total object spectrum width). With exposures of $\mathrm{Hg}$ and $\mathrm{Ne}$ lamps taken immediately after the science exposures, the spectra were wavelength-calibrated. We checked the accuracy of the wavelength calibration using sky emission lines, and find typical deviations of $1 \AA$ for the blue range and $0.5 \AA$ for the red. The effective spectral resolution, derived by measuring the width of the arc lines is $3.5 \AA F W H M$ (corresponding to an instrumental dispersion about $90 \mathrm{~km} \mathrm{~s}^{-1}$ at $5007 \AA$ and $70 \mathrm{~km} \mathrm{~s}^{-1}$ at $\mathrm{H} \alpha$ wavelength). The continuum-lamp exposure was also used to determine the response of the instrument for each fiber and wavelength (the so-called flat spectra). We used these flat spectra to homogenize the response of all

\footnotetext{
${ }^{2}$ IRAF is distributed by the National Optical Astronomy Observatories.
}

the fibers. For the standard star observations, we co-added the spectra of the central fibers and compared the one-dimensional standard star spectrum with table values to create a sensitivity function. The spectra were flux calibrated using IRAF.

The reduced spectra were contained in a data cube for each object and spectral range and they were sky-subtracted and corrected for the effect of DAR using the R3D package (Sánchez 2006).

\section{Results}

\subsection{Line measurement and $\mathrm{H} \alpha$ maps}

We fitted each emission-line profile on the extracted onedimensional spectra of each spaxel using a Gaussian function over the local position of the continuum. We used an automatic routine based on the IRAF task splot to measure the flux of most of the emission lines. For the low $S / N$ emission-lines (e.g., [OIII] $4363 \AA$ ) and those lying close to the border of the CCD (e.g., [OII] $3727 \AA$ for HS 0128+2832 and Mrk 930), the fitting procedure was repeated using a manual measurement.

The measured flux of the Balmer emission lines is sometimes underestimated because of the presence of an underlying stellar continuum (e.g. Diaz 1988). For objects showing high $E W(\mathrm{H} \beta)$ values $(223 \AA$ in HS $0128+2832,230 \AA$ in HS $0837+4717$, and $93 \AA$ in Mrk 930), the effect of the stellar absorption is negligible due to the low typical values of $1-2 \AA$ for the hydrogen line $E W \mathrm{~s}$ in absoprtion (see e.g. McCall et al. 1985). However, to be careful, we carried out an eye inspection, fiber-by-fiber, of the $\mathrm{H} \beta$ and $\mathrm{H} \gamma$ emission lines, and we did not find any apparent stellar absorption feature (e.g., wings of absorption lines) underlying these emission lines.

We calculated the statistical error of the line fluxes, $\sigma_{l}$, using the expression $\sigma_{l}=\sigma_{\mathrm{c}} N^{1 / 2}[1+E W / N \Delta]^{1 / 2}$ (see Pérez-Montero \& Díaz 2003) where $\sigma_{c}$ represents a standard deviation in a box centered close to the measured emission line, $N$ is the number of pixels used in the measurement of the line flux, $E W$ is the equivalent width of the line, and $\Delta$ is the wavelength dispersion in $\AA /$ pixel. This expression takes the error in the continuum and the photon counts statistics of the emission line into account. The error measurements were performed on the extracted onedimensional spectra. These associated errors are quite different depending on the observed object. As a result, the average relative error of $\mathrm{H} \beta$ is $10 \%$ in HS $0128+2832$ and HS $0837+4717$, but only of $5 \%$ in Mrk 930. Besides, to minimize errors in the ratios between a certain emission line and $\mathrm{H} \beta$, we always took first its ratio in relation to the closest hydrogen emission line (i.e. $\mathrm{H} \alpha$ in the case of $\left[\mathrm{NII}_{\mathrm{II}}\right]$ and $\left[\mathrm{S}_{\mathrm{II}}\right]$ ), and then we renormalize it using the corresponding theoretical Balmer ratio (i.e. at the electron temperature reported in the long-slit observations for each object). We checked that the variation in this temperature across the field of view of the instrument does not introduce errors in the theoretical ratio higher than those associated with the flux of the emission lines.

The $\mathrm{H} \alpha$ emission line maps (continuum subtracted and extinction corrected) are shown in Fig. 3. In the same figure, the contours in gray solid line show the isophotes in units of $12.5 \%$ times the emission of the maximum of $\mathrm{H} \alpha$ in each galaxy. In the panel of Mrk 930, we also show the contours extracted from the HST-ACS image shown in Fig. 2. Although the combined low spatial resolution of the instrument $\left(1^{\prime \prime} /\right.$ spaxel $)$ and poor seeing during the observing night $\left(\sim 2^{\prime \prime}\right)$ do not allow precise analysis of the morphology of the galaxies, it is seen that in HS $0128+2832$ and HS $0837+4717$, the central compact burst dominates the 

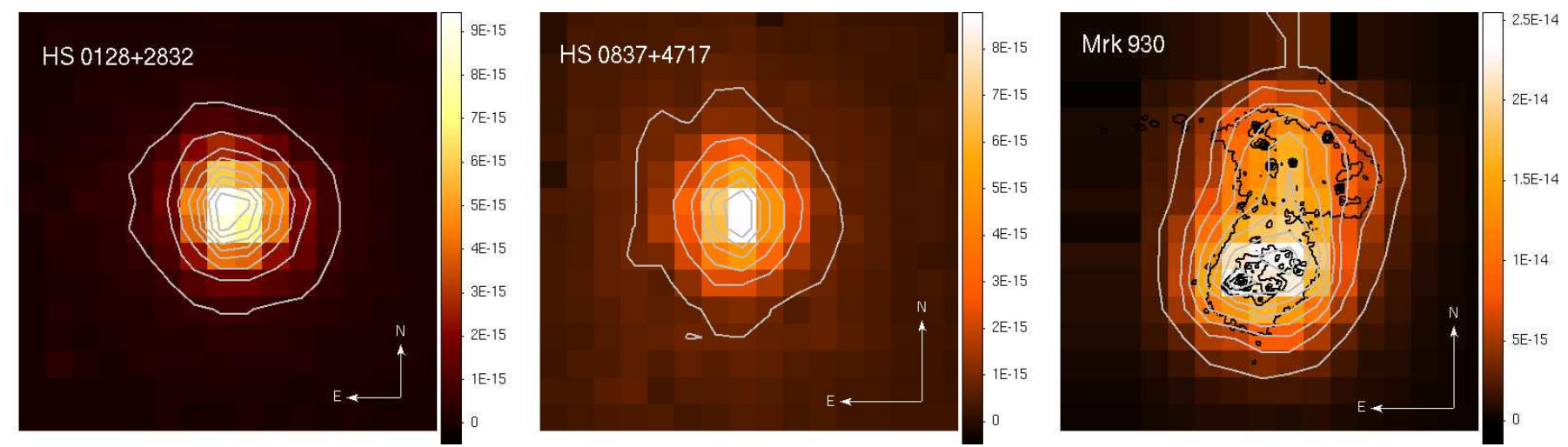

Fig. 3. Extinction-corrected H $\alpha$ maps of HS $0128+2832$, HS $0837+4717$, and Mrk 930 from left to right. The gray solid line contours show the isophotes in units of $12.5 \%$ of the maximum of $\mathrm{H} \alpha$. In the Mrk 930 image, black solid lines represent the contours extracted from the ACS-HST UV image shown in Fig. 2, which represent the position of the brightest super star clusters. In all images, each spaxel has 1" of resolution, north points up and east to the left. Units are in erg/s/ $/ \mathrm{cm}^{2} / \AA$.

emission of the galaxy, while Mrk 930 displays a more extended distribution of the star formation in agreement with what is observed in the UV image (see Fig. 2).

\subsection{Statistical distributions and integrated spectra}

Line intensities with their corresponding errors measured for each spaxel were used to obtain the maps of several physical properties and chemical abundances. Only those emission lines with $S / N>5$ were considered. In order to extract statistical information about the behavior of the various derived physical properties and chemical abundances, we also provide their distribution histograms, taking different regions into account around the $\mathrm{H} \alpha$ peak intensity, $\mathrm{F}(\mathrm{H} \alpha)_{\text {peak }}$. Hereafter in all histograms, dark blue bars represent the spaxels for which $\mathrm{F}(\mathrm{H} \alpha)$ $>25 \% \cdot \mathrm{F}(\mathrm{H} \alpha)_{\text {peak }}$ and light blue bars, those where $\mathrm{F}(\mathrm{H} \alpha)>$ $12.5 \% \cdot \mathrm{F}(\mathrm{H} \alpha)_{\text {peak }}$. Finally, white bars represent the distribution of the corresponding physical-chemical property in all spaxels with enough $S / N$ over the whole IFU area. In Table 3, we show the total $\mathrm{H} \alpha$ extinction-corrected fluxes in logarithm units, as measured in each of these regions for the three studied galaxies. The ratios between the $\mathrm{H} \alpha$ fluxes measured in the whole field of view of the IFU and the fluxes measured in long-slit observations, listed in Table 1, can be considered as aperture correction factors, giving an estimate for the $\mathrm{H} \alpha$ flux lost when using long-slit observations. In our cases, this gives ratios of 4.6 in HS $0128+2832,4.4$ in HS 0837+4717, and 6.9 in Mrk 930. In the case of Mrk 930, this factor is almost three times the aperture correction factor estimated by Guseva et al. (2000).

To ease the comparison between the studied distributions and the position of each defined region, we overplotted the corresponding $\mathrm{H} \alpha$ galaxy contours in all the maps, with the exception of Mrk 930, for which we used the contours from the ACS image, with better spatial resolution, aiding identification of the individual knots.

To ascertain whether a physical-chemical property can be considered as homogeneous across the IFU area, we followed a statistical criterion. We plotted the spaxel distribution of a given property in each region of each galaxy and studied whether that distribution can be fitted by a normal function. As a firstorder approximation, it can be assumed that the measured variations around the mean value of the distribution mainly have a statistical origin, so that a normal distribution of measurements/derivations can be considered. With this aim, a Lilliefors test (Lilliefors 1967) was carried out for each of the distributions of the derived properties and abundances in each of the regions defined above. For this test, the null hypothesis is that the data come from an unspecified normal distribution. We then assumed that if the null hypothesis cannot be rejected at the $10 \%$ of significance level, the distribution is considered as normal. In that case, the fitted Gaussian is plotted in the corresponding histogram. The number of spaxels used to do this test in each region of the IFU, with the mean value, the dispersion of the fitted Gaussian, and the significance level are listed in Table 3 and the corresponding values are noted in bold font. Otherwise (i.e. if the significance level is lower than 10\%), the mean and dispersion values are those of the distribution not fitted.

A comparative analysis between the distribution of the derived properties in the spaxels and their values in the integrated spectra was done by co-adding of the emission of those spaxels located in the central region (i.e. presenting $\mathrm{H} \alpha$ emission higher than $25 \%$ of the intensity in the peak of each galaxy). In contrast to the statistical distribution method described above, this procedure gives a flux-weighted average of the properties of the galaxy, and it is more consistent to establish a comparison with long-slit spectra. In the case of Mrk930, we extracted two spectra: one around the southern brightest knot and a second one close to the northern knots. The relative dereddened emission lines and the derived properties for each of the integrated spectra, as described in the sections below, are summarized in Table 4.

\subsection{Reddening correction and $c(H \beta)$ maps}

For each fiber spectrum we derived its corresponding reddening coefficient, $c(\mathrm{H} \beta)$, using the value of the Balmer decrement derived from $\mathrm{H} \alpha / \mathrm{H} \beta, \mathrm{H} \gamma / \mathrm{H} \beta$, and $\mathrm{H} \delta / \mathrm{H} \beta$ flux emission-line ratios, as compared to the theoretical values expected for recombination case B from Storey \& Hummer (1995) at the electron density and temperature reported in the literature using long-slit observations, and applying the extinction law given by Cardelli et al. (1989) with $R_{V}=3.1$. Thus, the fluxes of the emission lines for each fiber were corrected for extinction using their corresponding $c(\mathrm{H} \beta)$ values.

The distribution functions and the maps of the reddening constants, $c(\mathrm{H} \beta)$, are shown in Fig. 4 along with the same $\mathrm{H} \alpha$ and UV (in the case of Mrk 930) contours described above. The typical errors of the derived reddening constants are 0.2 in the three objects. A Gaussian fits the distribution of the three 
Table 3. Results of the Lilliefors test and the Gaussian fits to the histograms of the derived physical conditions and chemical abundances in the three studied objects for different subsets of spaxels.

\begin{tabular}{|c|c|c|c|c|c|c|c|c|c|c|}
\hline \multirow{2}{*}{$\begin{array}{l}\text { Object } \\
\text { Region }\end{array}$} & & \multicolumn{3}{|c|}{ HS $0128+2832$} & \multicolumn{3}{|c|}{ HS $0837+4717$} & \multicolumn{3}{|c|}{ Mrk 930} \\
\hline & & 1 & 2 & 3 & 1 & 2 & 3 & 1 & 2 & 3 \\
\hline $\log \mathrm{F}(\mathrm{H} \alpha)\left(\mathrm{erg} / \mathrm{s} / \mathrm{cm}^{2} / \AA\right)$ & & -13.00 & -12.83 & -12.57 & -12.84 & -12.69 & -12.42 & -12.06 & -11.95 & -11.87 \\
\hline \multirow[t]{4}{*}{$c(\mathrm{H} \beta)$} & Spaxels & 13 & 30 & 55 & 18 & 40 & 77 & 42 & 73 & 157 \\
\hline & Mean & 0.37 & 0.40 & 0.39 & 0.70 & 0.79 & 0.87 & 0.48 & 0.46 & 0.51 \\
\hline & Dispersion & 0.25 & 0.28 & 0.29 & 0.34 & 0.24 & 0.23 & 0.24 & 0.24 & 0.31 \\
\hline & Sign. $(\%)$ & $<10$ & $<10$ & $<10$ & 71 & 80 & 49 & $<10$ & $<10$ & $<10$ \\
\hline \multirow{4}{*}{$\log ([$ OII]/[OIII]) } & Spaxels & 14 & 29 & 55 & 17 & 28 & 37 & 47 & 66 & 143 \\
\hline & Mean & -0.97 & -0.96 & -0.80 & -0.94 & -0.88 & -0.83 & -0.31 & -0.33 & -0.22 \\
\hline & Dispersion & 0.10 & 0.20 & 0.41 & 0.21 & 0.21 & 0.26 & 0.18 & 0.18 & 0.21 \\
\hline & Sign. (\%) & $<10$ & $<10$ & $<10$ & $<10$ & 13 & 59 & 38 & 64 & $<10$ \\
\hline \multirow[t]{4}{*}{$n\left(\left[\mathrm{~S}_{\mathrm{III}}\right]\right)\left(\mathrm{cm}^{-3}\right)$} & Spaxels & - & - & - & - & - & - & 38 & 67 & 107 \\
\hline & Mean & - & - & - & - & - & - & 101 & 213 & 271 \\
\hline & Dispersion & - & - & - & - & - & - & 135 & 275 & 296 \\
\hline & Sign. (\%) & - & - & - & - & - & - & $<10$ & $<10$ & $<10$ \\
\hline \multirow[t]{4}{*}{$t([\mathrm{OIII}])\left(10^{4} \mathrm{~K}\right)$} & Spaxels & 13 & 22 & 22 & 10 & 12 & 13 & 39 & 52 & 52 \\
\hline & Mean & 1.35 & 1.37 & 1.37 & 1.64 & 1.64 & 1.62 & 1.26 & 1.29 & 1.29 \\
\hline & Dispersion & 0.10 & 0.19 & 0.19 & 0.25 & 0.24 & 0.24 & 0.18 & 0.21 & 0.21 \\
\hline & Sign. (\%) & $<10$ & $<10$ & $<10$ & 22 & 37 & 43 & 11 & 15 & 15 \\
\hline $12+\log (\mathrm{O} / \mathrm{H})$ & Spaxels & 9 & 21 & 21 & 10 & 12 & 13 & 35 & 47 & 47 \\
\hline \multirow{3}{*}{ (direct method) } & Mean & 8.00 & 7.99 & 7.99 & 7.83 & 7.82 & 7.85 & 8.18 & 8.15 & 8.15 \\
\hline & Dispersion & 0.09 & 0.10 & 0.10 & 0.15 & 0.14 & 0.18 & 0.16 & 0.18 & 0.18 \\
\hline & Sign. (\%) & $<10$ & $<10$ & $<10$ & 41 & 15 & 11 & 55 & 32 & 32 \\
\hline $12+\log (\mathrm{O} / \mathrm{H})$ & Spaxels & 14 & 29 & 50 & 16 & 27 & 32 & 41 & 79 & 79 \\
\hline \multirow[t]{3}{*}{ (R23-P method) } & Mean & 8.01 & 7.99 & 7.96 & 7.88 & 7.90 & 7.92 & 8.18 & 8.14 & 8.14 \\
\hline & Dispersion & 0.09 & 0.11 & 0.28 & 0.18 & 0.19 & 0.23 & 0.16 & 0.19 & 0.19 \\
\hline & Sign. (\%) & $<10$ & 25 & $<10$ & 98 & 17 & 18 & 13 & $<10$ & $<10$ \\
\hline $\log (\mathrm{N} / \mathrm{O})$ & Spaxels & 13 & 17 & 17 & 9 & 11 & 12 & 36 & 49 & 51 \\
\hline \multirow{3}{*}{ (direct method) } & Mean & -1.19 & -1.17 & -1.17 & -0.82 & -0.81 & -0.82 & -1.43 & -1.44 & -1.43 \\
\hline & Dispersion & 0.11 & 0.16 & 0.16 & 0.16 & 0.15 & 0.14 & 0.13 & 0.19 & 0.18 \\
\hline & Sign. (\%) & 72 & 67 & 67 & 82 & 83 & 57 & 22 & 12 & 38 \\
\hline $\log (\mathrm{N} / \mathrm{O})$ & Spaxels & 15 & 25 & 25 & 17 & 28 & 34 & 47 & 84 & 124 \\
\hline \multirow[t]{3}{*}{ (N2O2 method) } & Mean & -1.18 & -1.12 & -1.12 & -0.85 & -0.80 & -0.79 & -1.36 & -1.43 & -1.41 \\
\hline & Dispersion & 0.25 & 0.27 & 0.27 & 0.26 & 0.28 & 0.29 & 0.24 & 0.17 & 0.11 \\
\hline & Sign. (\%) & $<10$ & $<10$ & $<10$ & $<10$ & 18 & 27 & 28 & $<10$ & $<10$ \\
\hline
\end{tabular}

Notes. The number of spaxels considered for the statistics for each region is done. Sign. stands for the significance level of the null hypothesis in the Lilliefors test. If the null hypothesis cannot be rejected with more than $10 \%$ confidence, then the Gaussian fit is assumed and the corresponding mean and dispersion values are taken (in bold font). Otherwise, the mean and dispersion are those of the distribution not fitted.

considered regions of HS $0837+4717$ with a mean value quite similar to what is found in the integrated spectrum $[c(\mathrm{H} \beta)=0.80]$. On the contrary, in HS $0128+2832$ none of the three distributions are fitted by a Gaussian function, and the derived value for the integrated spectrum of the inner-most region is much lower (0.14) than the mean of the distribution for the same region (0.37), which could indicate a lower extinction in the $\mathrm{H} \alpha$ peak region. This behavior also appears in the histogram of Region 1 for this galaxy, where a double-peak structure can be seen. In Mrk 930 no Gaussian fits any of the three distributions, which are centered at low values of the reddening, although they can be seen some spaxels with reddening $c(\mathrm{H} \beta)>1.0$ breaking the normal distribution in all the regions. By visual inspection of the corresponding $c(\mathrm{H} \beta)$ map, these appear above all in the south west part of the galaxy, somehow resembling the ring-like structure shown by Adamo et al. (2011).

\subsection{Ionization structure and excitation}

Diagnostic diagrams (BPT, Baldwin et al. 1981) are frequently used to distinguish between different ionization sources of the surrounding gas emitting the detected bright emission lines. Active galactic nuclei (AGNs), shocks or massive stars can be selected by comparing the ratios of low excitation-to-Balmer emission lines, such as [NII] at $6584 \AA$, [SII] at $6717 \AA, 6731 \AA$, or [OI] at $6300 \AA$, to $\mathrm{H} \alpha$ in relation to a high excitation-toBalmer emission line ratios, like [OIII] at $5007 \AA$ to $\mathrm{H} \beta$. In all studies about our three-galaxy sample, their integrated spectra show a clear star-forming ionization origin in all these three diagrams. Nevertheless, the analysis of the individual spaxels in our observations reveals unexpected behavours when we look at the $[\mathrm{NII}] / \mathrm{H} \alpha$ vs. $[\mathrm{O}$ III $] / \mathrm{H} \beta$ diagram, shown in Fig. 5. In order to be compared with our observations, we took as a reference the theoretical curve given by Kewley et al. (2001) and the empirical fitting described by Kauffmann et al. (2003). Following Kewley et al. (2006), the region between these two lines could correspond to galaxies with a composite source of ionization, partially from star formation and partially from X-rays, while the area under the curves could correspond to pure star-forming regions and the area over the curves to pure AGNs. LINERs would lie in the low region at right. As can be seen, for all the three objects, most of the spaxels in Region 1 and the integrated spectrum lie in the SF region, although the points are close to the Kewley's curve for HS $0837+4717$. Nevertheless, a large fraction of the spaxels in Regions 2 and 3 (i.e. those in the outer regions of the galaxies) lie both in the composite and AGN regions in HS $0128+2832$ 
E. Pérez-Montero et al.: A PMAS study of high N/O BCDs

Table 4. Dereddened emission line intensities, relative to $\mathrm{I}(\mathrm{H} \beta)=1000$, and physical properties and oxygen and nitrogen chemical abundances with their corresponding propagated errors, as derived from the integrated spectra of the studied galaxies.

\begin{tabular}{|c|c|c|c|c|}
\hline & HS $0128+2832$ & HS $0837+4717$ & Mrk 930-S & Mrk 930-N \\
\hline$\lambda(\AA)$ & $I(\lambda)$ & $I(\lambda)$ & $I(\lambda)$ & $I(\lambda)$ \\
\hline 3727 [OII] & $788 \pm 116$ & $1045 \pm 87$ & $2672 \pm 43$ & $2532 \pm 58$ \\
\hline $4102 \mathrm{H} \delta$ & $270 \pm 9$ & $271 \pm 22$ & $277 \pm 10$ & $292 \pm 18$ \\
\hline $4340 \mathrm{H} \gamma$ & $455 \pm 11$ & $470 \pm 24$ & $484 \pm 11$ & $474 \pm 18$ \\
\hline 4363 [ÖIII] & $100 \pm 8$ & $149 \pm 12$ & $54 \pm 6$ & $40 \pm 5$ \\
\hline $4861 \mathrm{H} \beta$ & $1000 \pm 27$ & $1000 \pm 45$ & $1000 \pm 15$ & $1000 \pm 20$ \\
\hline 4959 [ОіII] & $2269 \pm 48$ & $2021 \pm 77$ & $1593 \pm 22$ & $1452 \pm 21$ \\
\hline 5007 [OIII] & $6962 \pm 139$ & $6064 \pm 195$ & $4817 \pm 59$ & $4441 \pm 65$ \\
\hline $6563 \mathrm{H} \alpha$ & $2811 \pm 66$ & $2774 \pm 102$ & $2830 \pm 47$ & $2849 \pm 53$ \\
\hline 6584 [NII] & $78 \pm 8$ & $152 \pm 10$ & $128 \pm 10$ & $136 \pm 20$ \\
\hline 6717 [SII] & $231 \pm 20$ & - & $257 \pm 10$ & $309 \pm 30$ \\
\hline $6731[\mathrm{SII}]$ & $176 \pm 20$ & - & $198 \pm 10$ & $215 \pm 20$ \\
\hline$c(\mathrm{H} \beta)$ & $0.14 \pm 0.05$ & $0.80 \pm 0.10$ & $0.43 \pm 0.07$ & $0.32 \pm 0.08$ \\
\hline $\log ([\mathrm{OII}] /[\mathrm{OIII}]$ & $-1.07 \pm 0.09$ & $-0.89 \pm 0.07$ & $-0.37 \pm 0.02$ & $-0.38 \pm 0.01$ \\
\hline$n([\mathrm{~S} I I])\left(\mathrm{cm}^{-3}\right)$ & $200_{-170}^{+260}$ & - & $<190$ & $32_{-19}^{+20}$ \\
\hline$t([\mathrm{OIII}])\left(10^{4} \mathrm{~K}\right)$ & $1.32 \pm 0.05$ & $1.69 \pm 0.10$ & $1.20 \pm 0.05$ & $1.11 \pm 0.05$ \\
\hline$t([\mathrm{OII}])\left(10^{4} \mathrm{~K}\right)^{3}$ & $1.12 \pm 0.08$ & $1.45 \pm 0.20$ & $1.16 \pm 0.05$ & $1.11 \pm 0.07$ \\
\hline$t\left(\left[\mathrm{~N}_{\mathrm{II}}\right]\right)\left(10^{4} \mathrm{~K}\right)^{a}$ & $1.25 \pm 0.03$ & $1.41 \pm 0.04$ & $1.19 \pm 0.03$ & $1.14 \pm 0.03$ \\
\hline $12+\log \left(\mathrm{O}^{+} / \mathrm{H}^{+}\right)$ & $7.09 \pm 0.24$ & $6.90 \pm 0.21$ & $7.69 \pm 0.10$ & $7.89 \pm 0.13$ \\
\hline $12+\log \left(\mathrm{O}^{2+} / \mathrm{H}^{+}\right)$ & $7.99 \pm 0.06$ & $7.67 \pm 0.07$ & $7.95 \pm 0.06$ & $8.01 \pm 0.07$ \\
\hline $12+\log (\mathrm{O} / \mathrm{H})$ & $8.04 \pm 0.08$ & $7.73 \pm 0.10$ & $8.14 \pm 0.05$ & $8.24 \pm 0.12$ \\
\hline $12+\log (\mathrm{O} / \mathrm{H})(\mathrm{R} 23-\mathrm{P})$ & 7.95 & 7.90 & 8.19 & 8.10 \\
\hline $12+\log \left(\mathrm{N}^{+} / \mathrm{H}^{+}\right)$ & $5.95 \pm 0.10$ & $6.14 \pm 0.05$ & $6.14 \pm 0.03$ & $6.22 \pm 0.03$ \\
\hline $\log (\mathrm{N} / \mathrm{O})$ & $-1.14 \pm 0.25$ & $-0.76 \pm 0.23$ & $-1.47 \pm 0.10$ & $-1.59 \pm 0.15$ \\
\hline $\log (\mathrm{N} / \mathrm{O})(\mathrm{N} 2 \mathrm{O} 2)$ & -1.14 & -0.98 & -1.41 & -1.50 \\
\hline
\end{tabular}

Notes. Mrk 930 has been divided between the brightest southern knot, and those situated in the northern region.
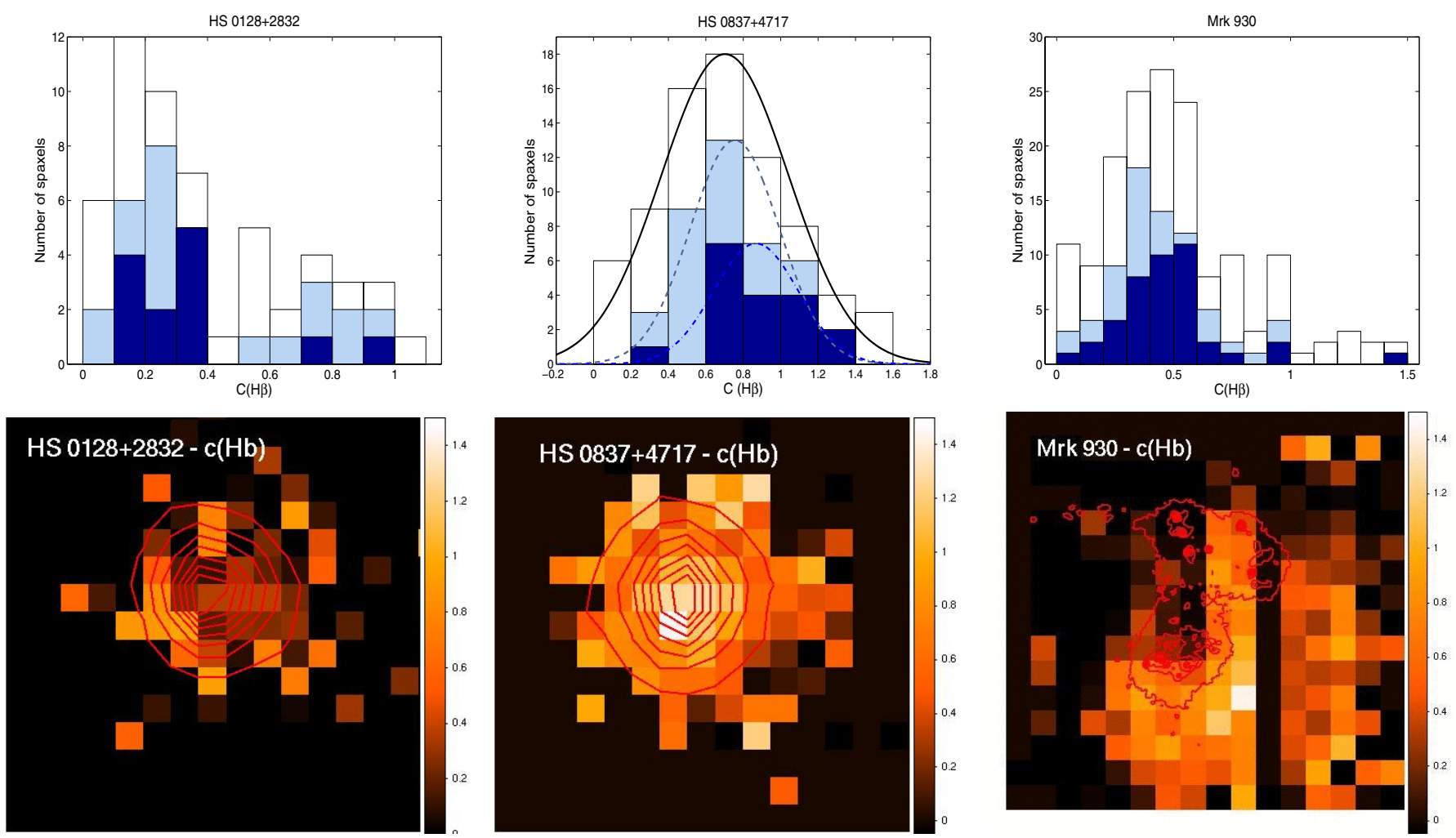

Fig. 4. Reddening constant maps of HS 0128+2832, HS 0837+4717, and Mrk 930 from left to right. The solid line contours are those described in Fig. 3. The histogram plots above represent the distribution of the extinction in different regions: dark blue bars represent the spaxels in Region 1 (spaxels with a $\mathrm{F}(\mathrm{H} \alpha)$ larger than $25 \%$ of the maximum), light blue bars, in Region 2 (spaxels with $\mathrm{F}(\mathrm{H} \alpha)$ larger than $12.5 \%$ of the maximum), and white bars, Region 3 (all the spaxels in the observed field with $S / N$ ). 

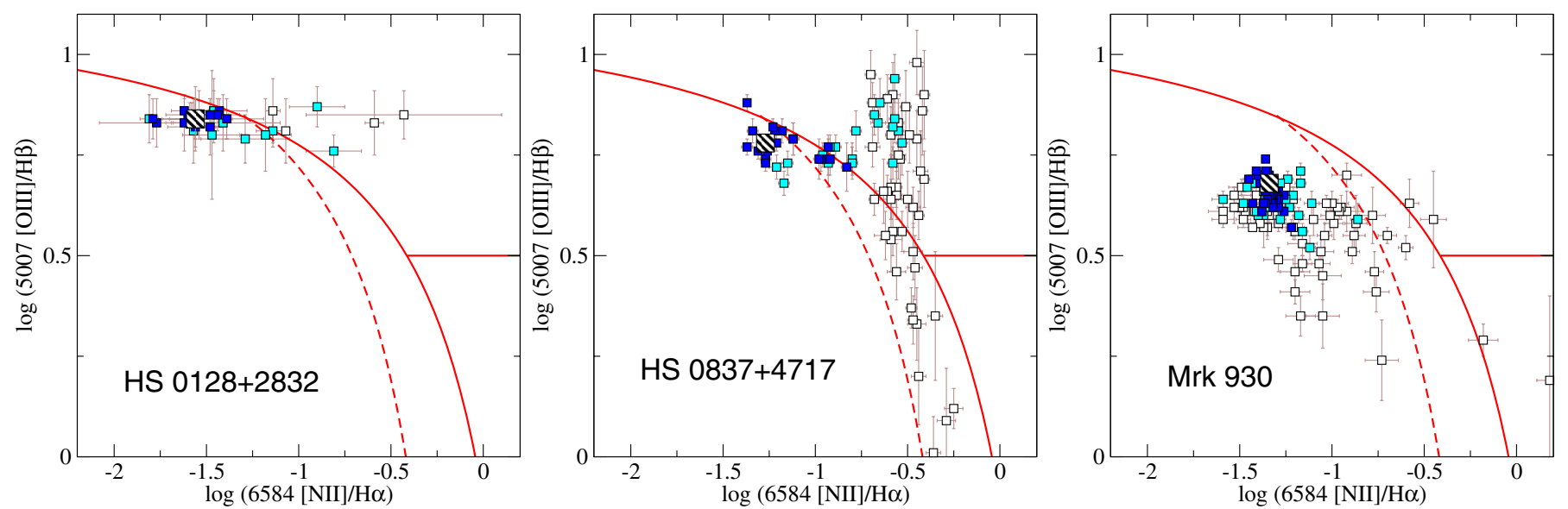

Fig. 5. Relation between $\log ([\mathrm{NII}] / \mathrm{H} \alpha)$ and $\log ([\mathrm{OIII}] / \mathrm{H} \beta)$. one of the so-called BPT diagrams for the individual spaxels of the three studied galaxies. Dark blue squares represent the spaxels in Region 1, the light blue, Region 2, and the white all the rest. Large stripped squares represent the result of the integrated spectrum of Region 1. The red dashed line represents the empirical curve defined by Kauffman et al. (2003) to separate star-forming galaxies from active galactic nuclei. The solid line represents the theoretical separation defined by Kewley et al. (2001).
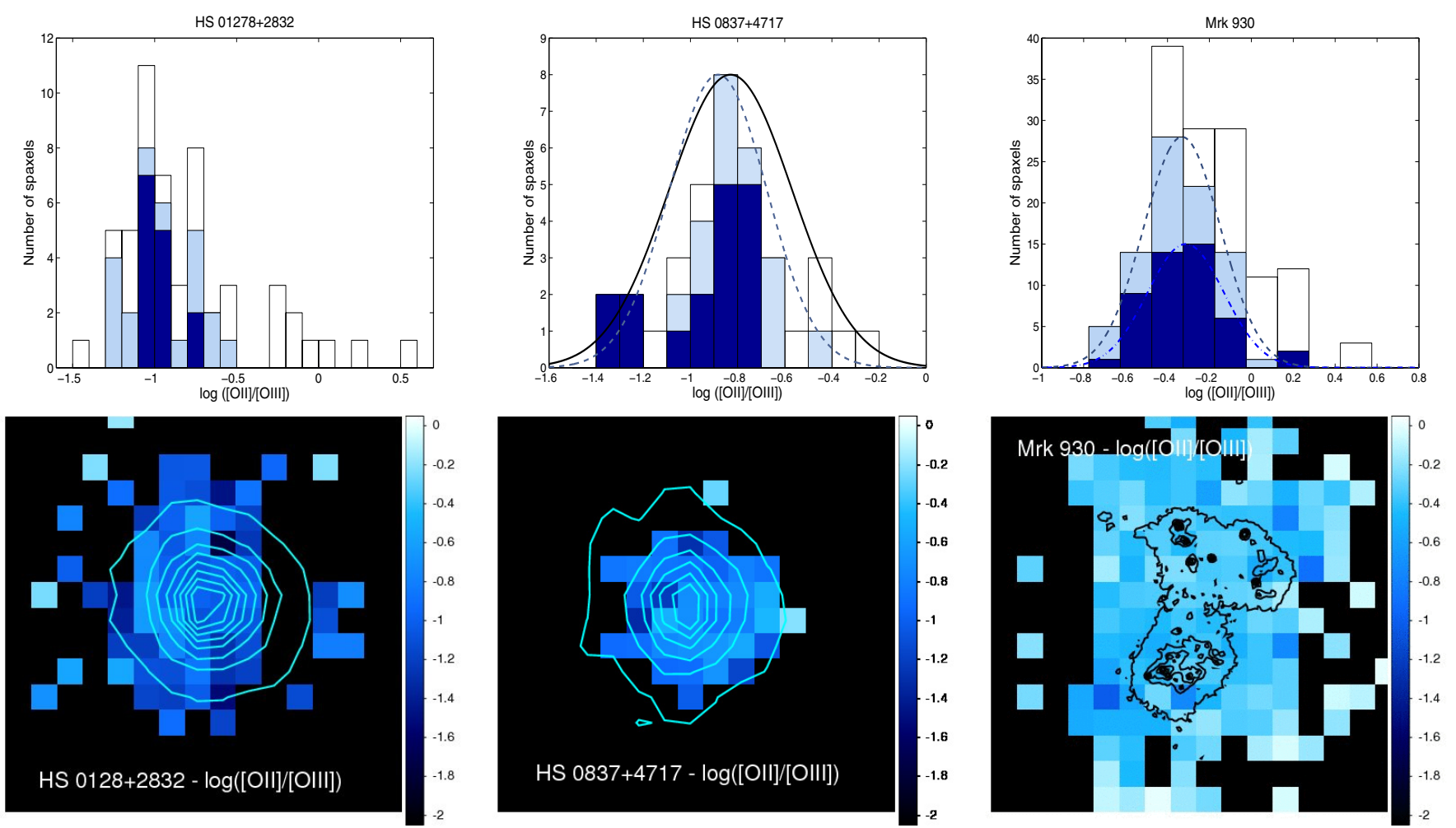

Fig. 6. Distribution histograms (top) and maps (bottom) of the excitation maps in terms of $\log ([\mathrm{OII}] /[\mathrm{OIII}])$ in HS 0128+2832, HS 0837+4717, and Mrk 930 from left to right. The solid lines show the same contours as in Fig. 3. Units are in dex. The distribution regions are the same as defined in Fig. 4.

and Mrk 930. For HS $0837+4717$, most of these spaxels lie in the AGN region.

The excitation of the ionized gas is studied by using the ratio of emission-line fluxes of [OII] at $3727 \AA$ and [OIII] at 4959, $5007 \AA$. Although this ratio depends somewhat on the effective equivalent temperature of the ionizing cluster and on the metallicity of the gas, it mainly depends on the ionization parameter (i.e. the ratio between the number of ionizing photons and particles, Díaz, 1998). In Fig. 6 we see the maps of the logarithm of this ratio and their corresponding distributions. The typical errors of this ratio range from 0.15 dex in HS $0128+2832$ and HS $0837+4717$ to only 0.03 dex in Mrk 930 . This ratio tends to be higher because more spaxels in the outer regions are considered, consistently with the larger distance to the ionizing central stellar cluster. In the case of Mrk 930, the Gaussian fits are found in the inner regions. In contrast, in HS $0837+4717$, the Gaussian fits only the outer regions, although this is possibly because there are fewer spaxels (i.e. for all the spaxels in the field of view with the required $S / N)$. For the three objects, the values in the integrated spectra are lower than the averages found in the histograms of the different regions, especially in HS $0837+4717$. In the case of Mrk 930, almost no difference has been found between the excitation of the southern and northern knots, which are in good agreement with the values found for the Gaussian fit of the innermost region. 

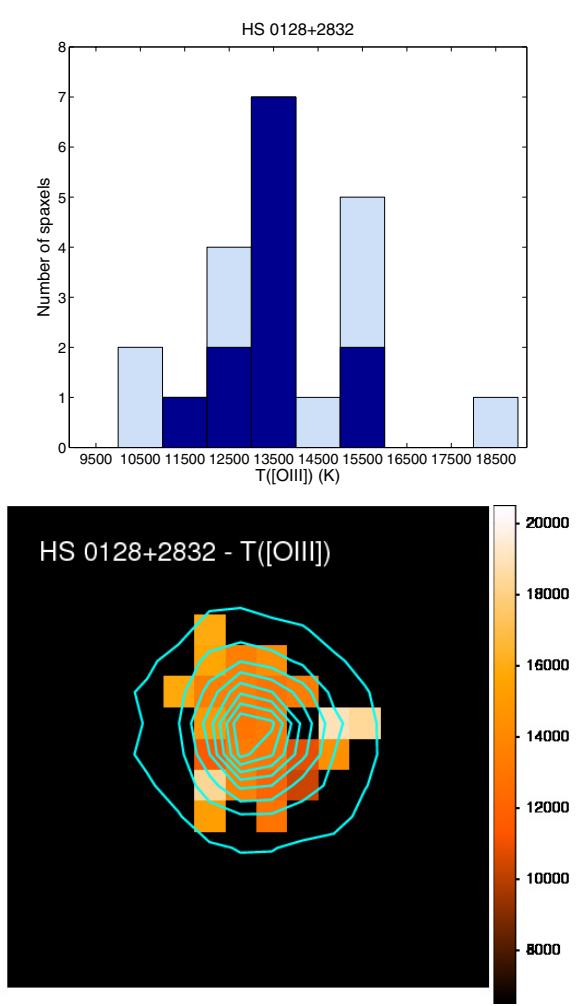
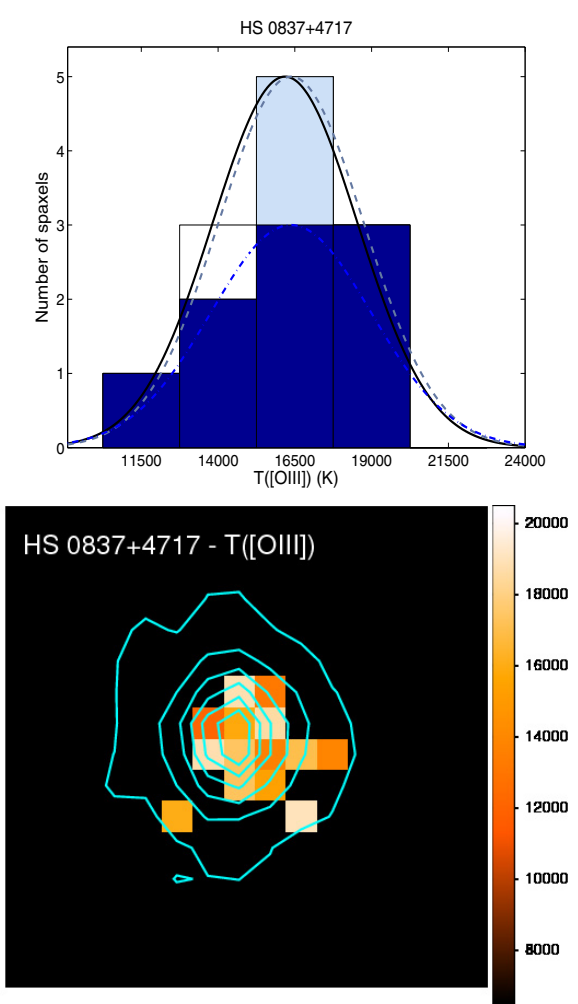
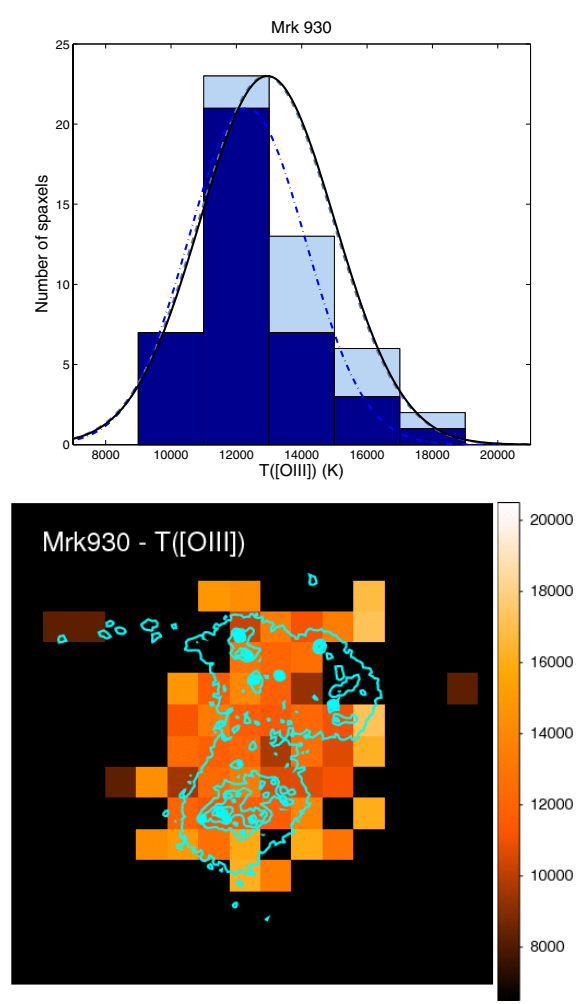

Fig. 7. Distribution histograms (top) and maps (bottom) of the electron temperature of [OIII] in HS 0128+2832, HS 0837+4717, and Mrk 930 from left to right respectively. The solid lines show the same contours as in Fig. 3. Units are in K. The distribution regions are the same defined in Fig. 4.

\subsection{Electron temperature and density}

Due to the low metallicity content of these galaxies, the cooling of the gas is not very efficient and the electron temperature is high, therefore, the electron temperature of [OIII] has been estimated in a large number of spaxels for our three galaxies by means of the flux ratio between the strong nebular emission-lines [OIII] 4959, $5007 \AA$ and the auroral weaker line at $4363 \AA$. To calculate the electron temperature $t([\mathrm{OIII}])$, we use the expressions from Hägele et al. (2006, 2008) that are based on the TEMDEN task under the IRAF nebular package. The distribution functions and the maps of this temperature in the three observed galaxies are shown in Fig. 7. The average error in the individual spaxels is much higher for HS 0128+2832, where it reaches $2000 \mathrm{~K}$. For HS $0837+4717$ and Mrk 930, the uncertainty is $\sim 700 \mathrm{~K}$. A Gaussian fit is found for the temperature values of all the regions in HS $0837+4717$ and in Regions 1 and 2 in Mrk 930, while no Gaussians were found for any region in HS $0128+2818$, although for this galaxy the mean value of $t$ ([OIII]) $(13200 \mathrm{~K})$ agrees within the errors with the value measured in the integrated spectrum. For HS $0837+4717$, an agreement is also found between the electron temperature of the integrated spectrum $(16900 \mathrm{~K})$ and the mean values derived in the three studied regions. For Mrk 930 the electron temperatures derived in the integrated spectra of both southern and northern knots are slightly lower (12000 and $11000 \mathrm{~K}$, respectively), but still within the dispersion of the corresponding Gaussian.

We derived the electron densities from the ratio between the [SII] emission lines at $6717,6731 \AA$ using the same TEMDEN routine. Only for Mrk 930 has it been possible to measure these lines with enough confidence in several spaxels. Only an upper limit to the density can be provided in the case of HS $0128+2832$, although a reliable estimate of the electron density has been made in the analysis of the integrated spectrum of the brightest region of this galaxy, but the errors are too high to achieve any precise determination. For HS $0837+4717$, the redshift of this galaxy placed the [SII] lines outside of the spectral range covered in this work. The distribution function and the map of the electron density in Mrk 930 are shown in Fig. 8. The average error in the spaxels is $50 \mathrm{~cm}^{-3}$. A Gaussian fit has not been found with enough confidence level for any of the analyzed regions. In fact, over the outer southern part of the galaxy, the electron density map displays high values, reaching up to $1700 \mathrm{~cm}^{-3}$, which is still below the critical density limit for collisional de-excitation. This makes the average density of all distributions higher in the outer regions and the density measured in the integrated spectrum of the southern knot to be slightly higher than in the northern knots.

\subsection{Oxygen and nitrogen chemical abundances}

In those spaxels with an estimate of the electron temperature, it is possible to derive chemical abundances following the direct method (i.e. the derivation of abundances using the relative emission flux of collisional-to-Balmer emission lines and the electron temperature). The $\mathrm{O}$ abundance was derived by calculating the abundances of the main states of ionization in the nebula: $\mathrm{O}^{2+}$ and $\mathrm{O}^{+}$, the most abundant under the excitation conditions of the gas in these objects. In both cases, we used expressions described in Hägele et al. $(2006,2008)$ based on the task IONIC under the IRAF nebular package. $\mathrm{O}^{2+}$ abundances were derived using the emission line intensities of 4959 and $5007 \AA$ relative to $\mathrm{H} \beta$ and the electron temperature of [OIII], which are available for a large number of spaxels.

In the case of $\mathrm{O}^{+}$abundances, we used the electron temperature of [OII] and the intensity of [OII] $3727 \AA$ relative to $\mathrm{H} \beta$. Since $t([\mathrm{OII}])$ was measured in any of the spaxels, we derived 

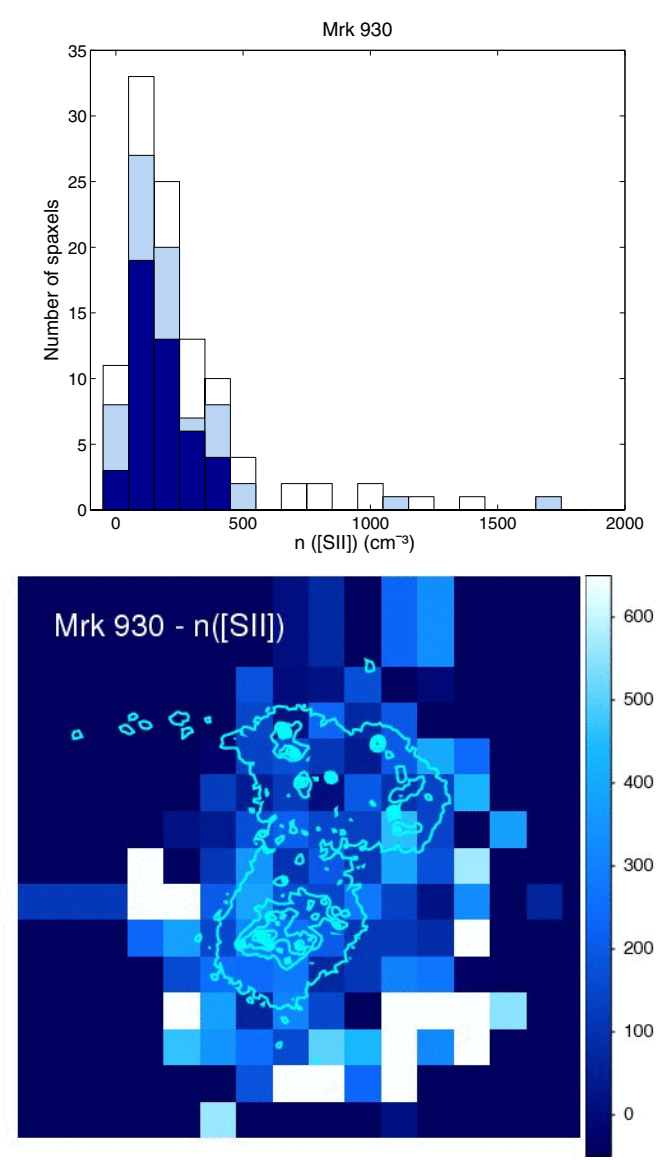

Fig. 8. Distribution histograms (top) and map (bottom) of the electron density from $\left[\mathrm{S}_{\mathrm{II}}\right]$ in Mrk 930 The solid lines show the same contours as in Fig. 3. Units are in $\mathrm{cm}^{-3}$. The distribution regions are the same defined in Fig. 4.

it from the available $t([\mathrm{OIII}])$ using the relations based on photoionization models proposed by Pérez-Montero \& Díaz (2003), which depend on the value of the electron density. In those spaxels without any estimate of the electron density (i.e. all in HS $0128+2832$ and HS $0837+4717$ and some of them in Region 3 of Mrk 930), we assumed a value of the electron density of $100 \mathrm{~cm}^{-3}$. The distribution functions and the maps of the total $\mathrm{O}$ abundance as derived using the direct method are shown in Fig. 9. Since the main source of uncertainty in this total abundance comes from the temperature, the average error is higher in HS $0128+2832$ (0.3 dex) than in HS $0837+4717$ and Mrk 930 ( 0.09 and 0.08 dex, respectively). As in the case of electron temperature a Gaussian fit is found in the three regions of HS $0837+4717$ and in Regions 1 and 2 of Mrk 930, but in none of HS $0128+2832$. The mean value of these distributions agree within the $1 \sigma$ dispersion range with the values derived from the integrated spectrum, although in the case of Mrk 930 there is a slight offset between the $\mathrm{O}$ abundance of the southern and northern knots, but it is within the observational errors.

A deeper study of the metallicity distribution of these galaxies was performed using strong-line methods. These do not depend on the previous determination of the electron temperature, and they can be applied in more spaxels. In the case of $\mathrm{O}$ abundance there are several indicators based on the intensity of the brightest lines of the spectrum. In our case, in order to be consistent with our previous estimation of the $\mathrm{O}$ abundance based on the direct method, we chose the calibration proposed by Pilyugin \& Thuan (2005), which is calibrated using objects whose metallicities were derived using electron temperature estimates. This method relies on calibrating the parameters $R 23$ and $P$, depending on [OII], [OIII], and $\mathrm{H} \beta$ emission-line fluxes. One of the main drawbacks of the strong-line methods based on R23 is the degeneracy of the parameter with $\mathrm{O}$ abundance (i.e. a same value of R23 leads to two different values of the O abundance). Nevertheless, since our three galaxies are metalpoor objects, the calibration proposed by Pilyugin \& Thuan for the low-metallicity regime was used. In Fig. 10, we show the distribution functions and the maps of the total $\mathrm{O}$ abundance in the three studied galaxies using this method. In all cases, the average errors in the spaxels of the three galaxies are lower than the uncertainty associated with the calibration of this parameter, which is 0.2 dex according to Pérez-Montero \& Díaz (2005). As for the $\mathrm{O}$ abundance derived using the direct method, in all three galaxies different Gaussian functions are fitted on different scales. In the case of the innermost region of HS $0837+4717$, the level of significance is very high (98\%), but it decreases substantially for the outer regions. In the case of HS $0128+2832$, a Gaussian fit is only found in Region 2. Finally, in Mrk 930, contrary to the direct method where Gaussians were found in Regions 1 and 2, a normal function is only fitted in Region 1. For the three objects, good agreement is found between the mean values of these distributions and their equivalents using the direct method in a lower number of spaxels and with the metallicity estimates from the integrated spectrum using the same parameters.

Nitrogen abundances were also calculated using both the direct method in those spaxels with an estimate of the electron temperature and using a strong-line method for all the other spaxels where a good measure of the bright emission lines exist. In the first case, we used the relative intensity of the [NII] $6584 \AA$ to $\mathrm{H} \beta$ and the electron temperature of [NII], estimated using the relation with $t$ ([OIII]) based on models described in Pérez-Montero \& Contini (2009). The N/O abundance ratio is then assumed to be equal to the $\mathrm{N}^{+} / \mathrm{O}^{+}$ratio. The distribution functions and the maps of this ratio are shown in Fig. 11. As for O total abundances derived from the direct method, the mean error of the $\mathrm{N} / \mathrm{O}$ in each spaxel is higher in HS 0128+2832 (0.4 dex) than in HS $0837+4717$ (0.2 dex) and Mrk 930 (0.16 dex). In contrast to all the other distributions in this work, Gaussian functions fit with a good level of confidence in all the regions of the three galaxies, in agreement with the values derived in the integrated spectra.

For the rest of spaxels, with no derivation of the temperature, the N/O can be estimated by resorting to the empirical calibration of the N2O 2 parameter proposed by Pérez-Montero \& Contini (2009). This parameter is based on the ratio of [NII] and [OII] emission lines, presenting a linear relation with the N/O. As previously, we used this method because it was calibrated using data with a direct estimation of the $\mathrm{N} / \mathrm{O}$, and it is consequently consistent with the $\mathrm{N} / \mathrm{O}$ derivation from the electron temperature. The distribution functions and the maps of the $\mathrm{N} / \mathrm{O}$ values using the strong-line method are shown in Fig. 12. The average errors are lower in all the spaxels than the uncertainty associated with the calibration of this parameter, which is 0.2 dex, according to Pérez-Montero \& Contini (2009). The mean values of the N/O distributions as derived from this strong-line method are compatible with the values found using the direct method. Nevertheless, the Gaussian fits appear only in the inner regions of HS $0837+4717$, and no Gaussians are found in any region for Mrk 930 and HS 0128+2818. 
E. Pérez-Montero et al.: A PMAS study of high N/O BCDs
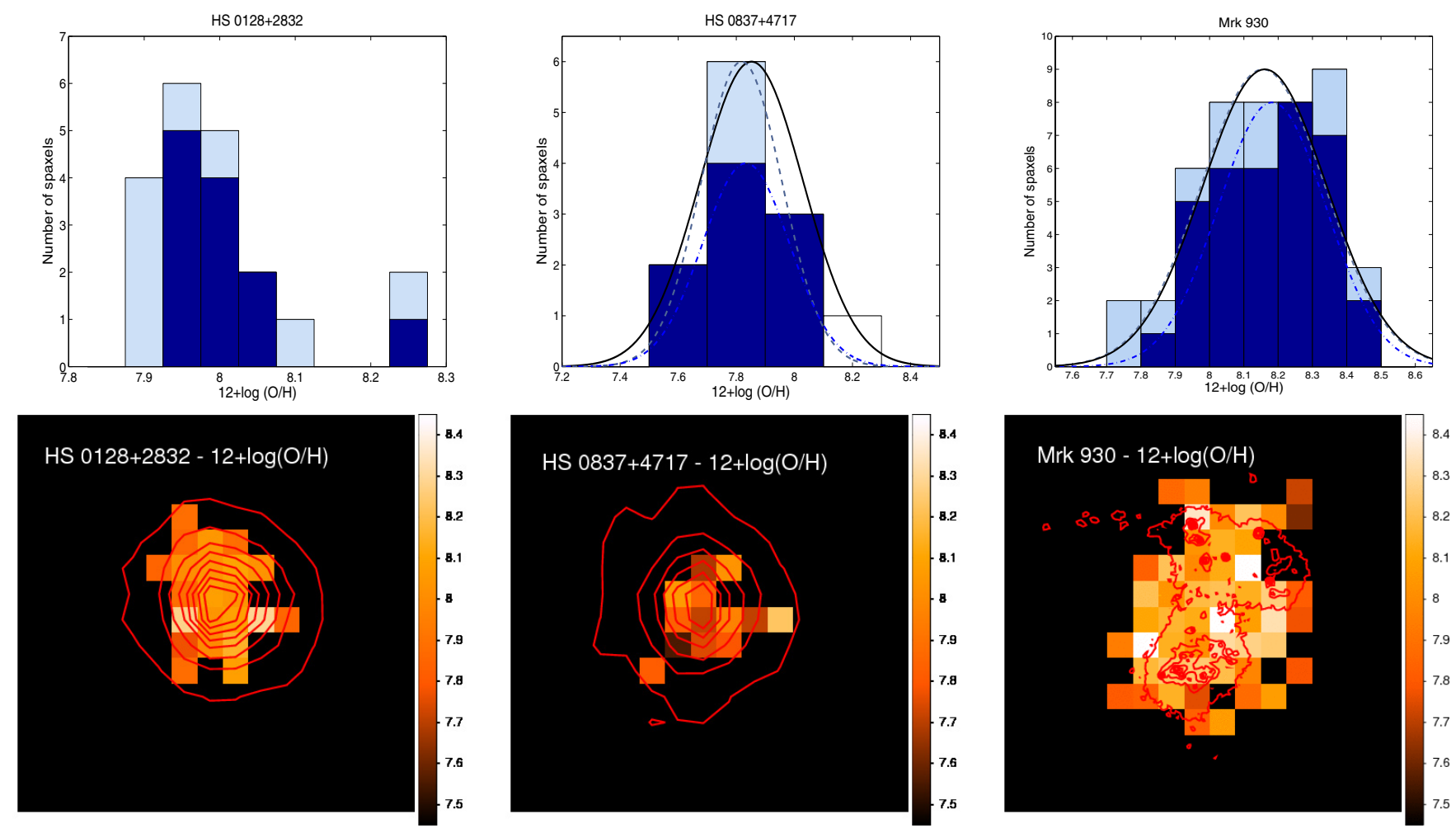

Fig. 9. Distribution histograms (top) and maps (bottom) of the total oxygen abundance, in terms of $12+\log (\mathrm{O} / \mathrm{H})$ calculated using the direct method, in HS $0128+2832$, HS $0837+4717$, and Mrk 930 from left to right. The solid lines show the same contours as in Fig. 3 . Units are in dex. The distribution regions are the same defined in Fig. 4.
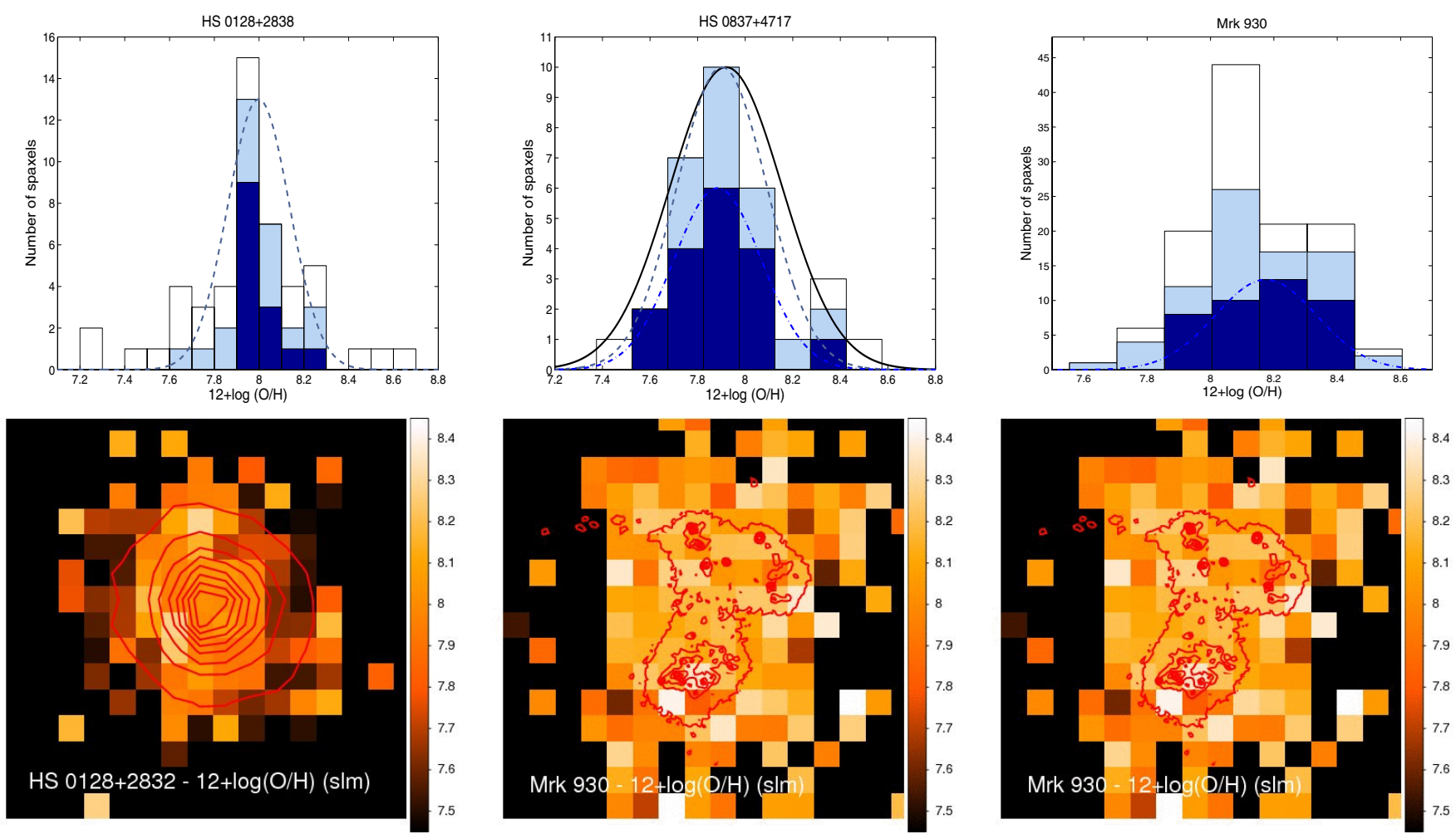

Fig. 10. Distribution histograms (top) and maps (bottom) of $12+\log (\mathrm{O} / \mathrm{H})$ calculated using the strong-line method based on the Pilyugin \& Thuan calibration, in HS $0128+2832$, HS $0837+4717$, and Mrk 930 from left to right. The solid lines show the same contours as in Fig. 3 . Units are in dex. The distribution regions are the same defined in Fig. 4. 

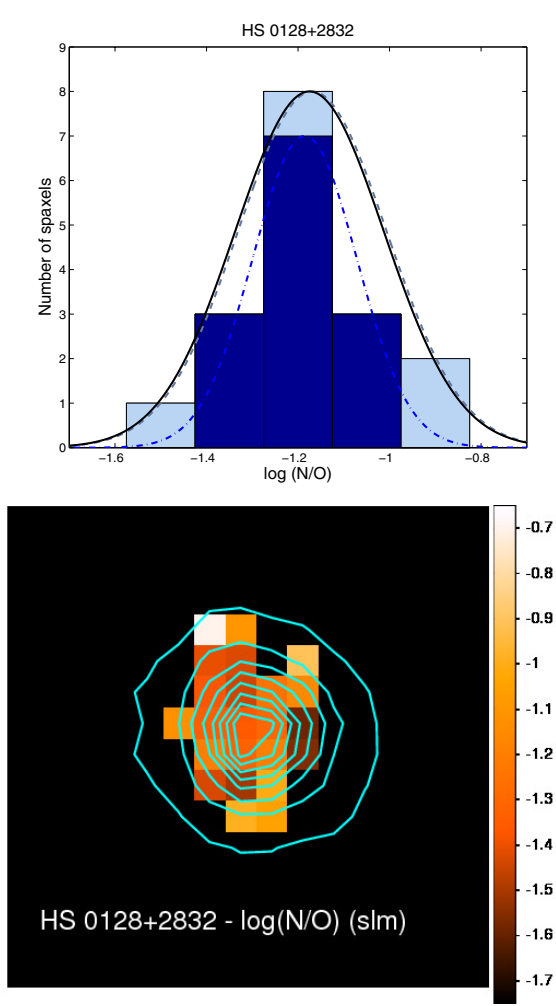
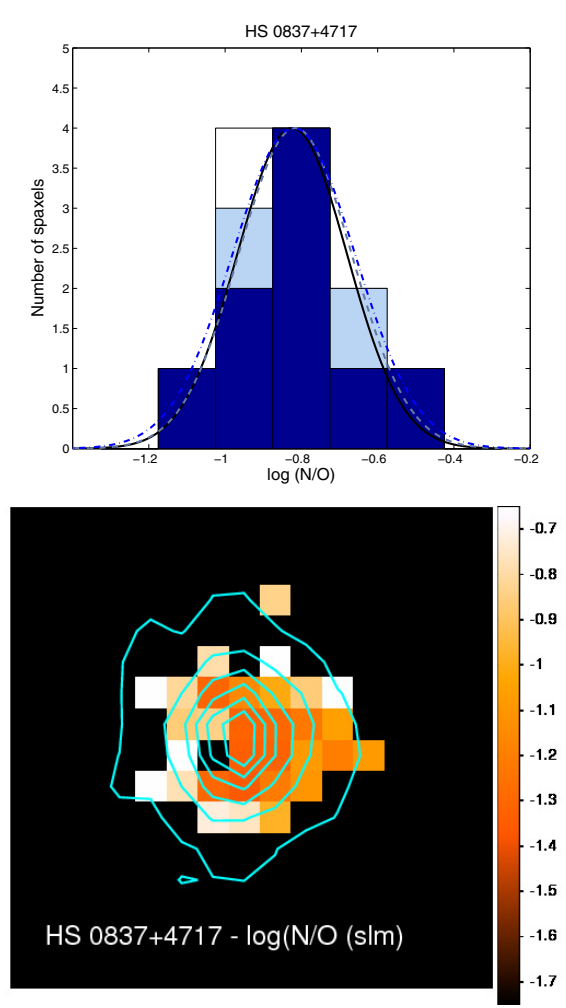
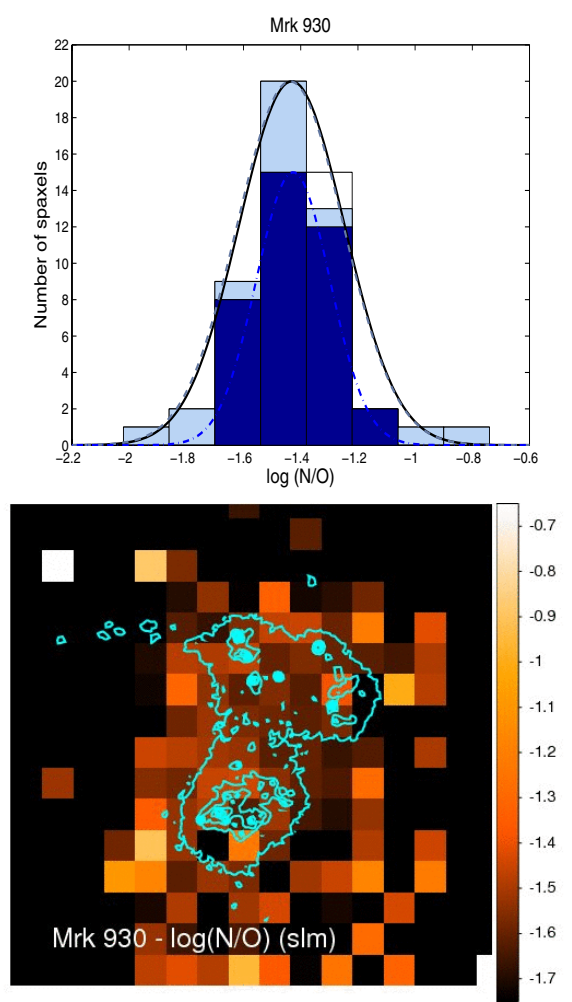

Fig. 11. Distribution histograms (top) and maps (bottom) of $\log (\mathrm{N} / \mathrm{O})$ calculated using the direct method, in HS 0128+2832, HS 0837+4717, and Mrk 930 from left to right. The solid lines show the same contours as in Fig. 3. Units are in dex. The distribution regions are the same defined in Fig. 4.
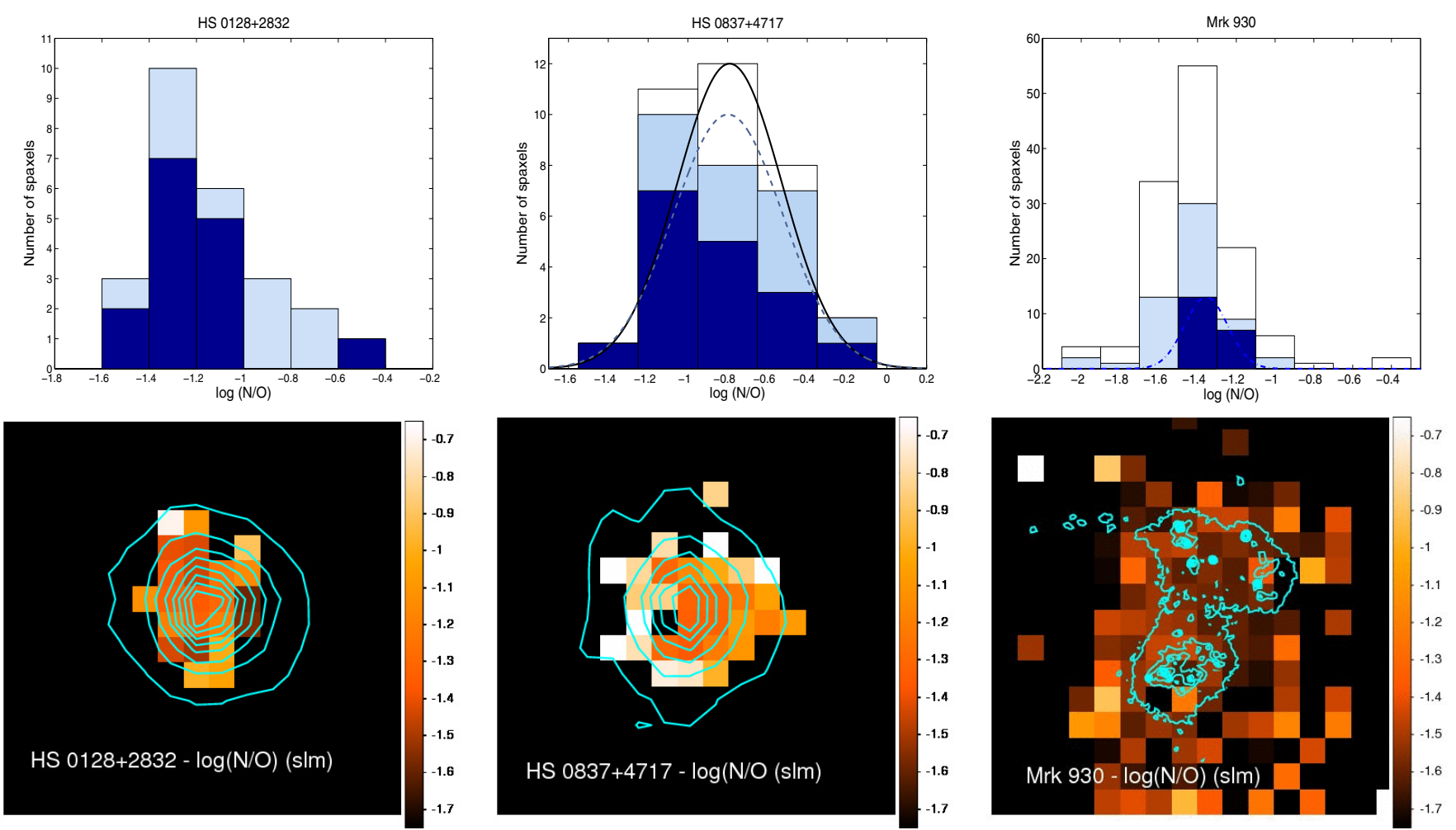

Fig. 12. Distribution histograms (top) and maps (bottom) of $\log (\mathrm{N} / \mathrm{O})$ calculated using the strong-line method based on the ratio [NII]/[OII], in HS $0128+2832$, HS $0837+4717$, and Mrk 930 from left to right. The solid lines show the same contours as in Fig. 3. Units are in dex. The distribution regions are the same defined in Fig. 4. 


\subsection{Kinematics of Mrk 930}

We studied the velocity field of the ionized gas by fitting a Gaussian to the $\mathrm{H} \alpha$ emission line in the three galaxies, but only Mrk 930 presents a rotation curve, as shown in the radial velocity map of Fig. 13. The spectral resolution of our data prevents us from deriving reliable velocity dispersions. The west side of the galaxy appears to be less disturbed than the east side; and a sharp velocity gradient in the north-south direction is visible in the eastern half of the map. This velocity field could be the result of an interaction system.

\section{Discussion}

\subsection{Spatial uniformity of the derived properties in the sample}

We studied the distribution of different physical properties and chemical abundances along the field of view of the PMAS instrument in some BCDs with different degrees of excess in their N/O in relation to the expected values for their metallicity regime. IFS allows a different approach to their study in contrast to integrated long-slit or fiber observations. In these cases, the integrated spectrum is a weighted mean of the different regions of the observed objects, whose statistical weights are the relative luminosities of the different regions. In contrast, IFS data can be analyzed by considering each observed region independent of their relative brightness. In this way, in the distributions of the physical properties and chemical abundances of the three studied BCDs in this work, the differences between the mean of the distributions in different regions and the values of the same quantities measured for the integrated spectrum are remarkable. These differences are illustrated well by the reddening constant distribution in the HS $0128+2832$ galaxy (see first panel of Fig. 4), whose integrated value is $c(\mathrm{H} \beta)=0.14$, while the mean value of the spaxel distribution is $c(\mathrm{H} \beta)=0.37$ in the innermost region. This difference is understood by inspecting the shape of the reddening constant distribution, where no Gaussian fit is found for any of the regions; on the contrary, an apparent double-peak distribution appears, with one of the maxima peaking at the same value found for the integrated spectrum and the other maximum peaking at the same value as the mean of all the data. Another example of this disagreement is found in the study of the density distribution of Mrk 930, which is too far to be fitted by a Gaussian. This is partially motivated by the presence of a high density structure visible in the south part of the galaxy and which is also seen in the histogram distribution plot (upper panel of Fig. 8). This structure is also responsible for the difference found in the electron density between the northern $\left(32 \mathrm{~cm}^{-3}\right)$ and southern $\left(<190 \mathrm{~cm}^{-3}\right)$ parts of the galaxy. Besides this, the mean values found for different regions of the same galaxy range between 101 and $271 \mathrm{~cm}^{-3}$, as we go from Region 1 (i.e. the innermost region, around the peak in $\mathrm{H} \alpha$ ) and Region 3 (i.e. all valid spaxels in the IFU), as seen in Table 3 . In the most part of the cases, this disagreement between the value found in the integrated spectrum and the mean value of the spaxels distribution appears when no Gaussian fit is found for this distribution, indicating local spatial variations, that cannot be understood only in terms of statistical fluctuations.

The histograms of the properties studied in different regions were also used to find out to what extent a certain property can be considered as homogeneous within the observational and statistical errors in different regions. This should allow us to find possible variations of the excitation as a function of the distance to the ionizing cluster, to find regions with excesses in electron

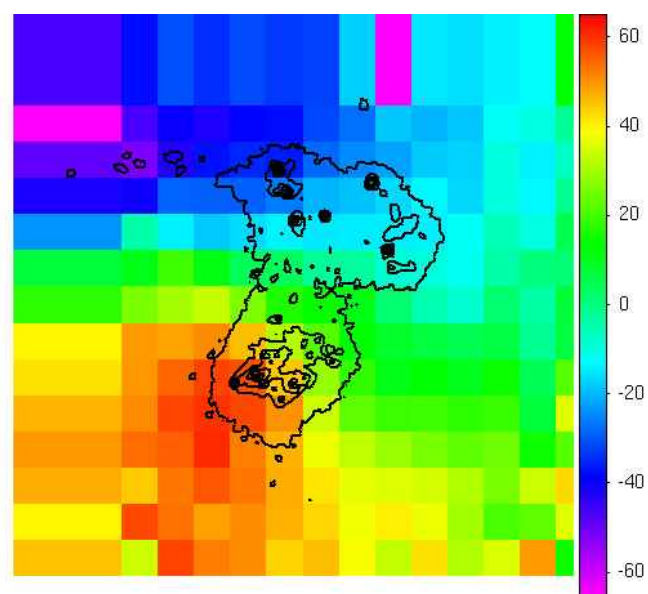

Fig. 13. Radial velocity of the ionized gas for the $\mathrm{H} \alpha$ emission line in Mrk 930. The solid lines show the same contours as in Fig. 3. Units are in $\mathrm{km} \mathrm{s}^{-1}$.

density or extinction, or to locate the chemical pollution in specified places in the observed field. However, the assumption of the hypothesis that a certain parameter can be considered as uniform if its spaxel distribution can be fitted by a Gaussian have two important limitations in this work, which must be taken into account. First, there is a sampling effect which means that, for some very compact objects, the number of spaxels considered valid can somehow be reduced. This is the case for the distributions of excitation and N/O, as derived using the $\mathrm{N} 2 \mathrm{O} 2$ parameter, in HS $0837+4717$ (middle panels of Figs. 6 and 12, respectively), in which the null hypothesis is rejected for Region 1. However, a Gaussian fit is found when the number of spaxels grow and Regions 2 and 3 are also considered. This possibly indicates that the rejection of the Gaussian fit hypothesis does not imply that a certain parameter is not uniform in a certain region if there are few points to be considered in the corresponding statistics. The second limitation comes from the poor seeing measured during the night of observations, which is worse in all cases than the working spatial resolution. In this case, serious doubts appear about the lack of correlation of the fluxes between adjacent spaxels. Nevertheless, the typical size of the regions where the uniformity was assumed is much greater in all cases than the typical value of the point spread function for the quoted seeing, and this is indicative of the validity of the results.

Another important fact that reinforces the assumption of uniformity in those cases where a Gaussian can be fitted is that the normal functions are normally found in more than a region in each galaxy. With the exception of the $\mathrm{O}$ abundance as derived from the R23-P method in HS $0128+2832$ and the N/O as derived from the $\mathrm{N} 2 \mathrm{O} 2$ method in Mrk 930, the rest of the physical properties and chemical abundances are fitted in two or even three defined regions in the field of view. This excludes the possibility in those cases of an artificial Gaussian distribution in a defined region of the space, as the fitting is found simultaneously in different and independent regions.

The results of these fittings listed in Table 3 indicate that in HS $0128+2832$ only the N/O as derived following the direct method can be fitted by a normal distribution. In contrast, in HS $0837+4717$, all the distributions can be fitted by a normal function at different regions, with the exception of the excitation and $\mathrm{N} / \mathrm{O}$ as derived from the $\mathrm{N} 2 \mathrm{O} 2$ method in Region 1, as explained above. Finally, in Mrk 930 only reddening and density distributions are not fitted by a Gaussian. In this case, the disturbed 
morphology of this galaxy is the most probable cause of these variations.

It is remarkable that in all the three galaxies the N/O as derived from the direct method can be considered as uniform on the spatial scales where it can be measured with enough precision, independently of the mean value of their distribution or the possible presence of WR stars. However, this is not the case for the same ratio when it is derived using an empirical calibrator, $\mathrm{N} 2 \mathrm{O} 2$ in this case, which does not appear as uniform in several regions of HS $0128+2832$ and Mrk 930. For total $\mathrm{O}$ abundances, the same discrepancy between the distributions derived from the direct method and from the empirical calibration of the R23 parameter given by Pilyugin \& Thuan (2005) is observed. In the case of Mrk 930, therefore, the $\mathrm{O}$ abundance distribution is well fitted by a Gaussian when it is derived using the direct method in Regions 1 and 2, but the Gaussian is only fitted in the innermost region when this abundance is derived using R23. Several limitations related to the calibration of these parameters can be considered when explaining this discrepancy, including the sample used to calibrate them, mostly data coming from integrated observations of star-forming knots, and the dependence of the calibrators on other functional parameters, such as the ionization parameter or the effective equivalent temperature (Pérez-Montero \& Díaz 2005), which are not uniform throughout a galaxy. Therefore, although the strong-line parameters allow the sampling of chemical abundances in more positions, they are less suitable to studying the homogeneity of these abundances than are other direct method-based techniques.

\subsection{Excitation variations and their implications on diagnostic diagrams}

One of the most interesting results of analyzing the spatial distributions of the properties of these three objects appears with the position of several spaxels in Fig. 5, which represents the $[\mathrm{NII}] / \mathrm{H} \alpha$ vs. $[\mathrm{OIII}] / \mathrm{H} \beta$ diagnostic diagram. As we see, the position of the integrated points and the spaxels belonging to Region 1 lie in the star-forming region, although the proximity of these points to both the theoretical and empirical curves is sensitive to the N/O (i.e. in HS $0128+2832$ and HS 0837+4717, which present the largest relative $\mathrm{N}$ abundance, these points are closer than in Mrk 930). Nevertheless, a fraction of the outer spaxels, which is greater in HS 0837+4717 and lower in Mrk 930, moves right in the diagrams so they can lie in the so-called composite region or even in the active galactic nuclei region. This effect is probably from the combination of two different effects: i) the high relative N/O that makes some points lie in these regions, as shown already by Pérez-Montero \& Contini (2009); and ii) the excitation structure of the galaxy, in which the [NII] emission line fluxes remain bright even at distances where the other emission lines are noticeably less bright. This makes the $[\mathrm{NII}] / \mathrm{H} \alpha$ higher in these regions, while $[\mathrm{OIII}] / \mathrm{H} \beta$ remains constant or decreases slightly. In this case the spectra of some positions of the field of view could be wrongly classified as AGNs. This effect was already observed by James et al. (2010) in the BCD Mrk 996 also using IFU observations.

\subsection{N/O excess and the presence of WR stars}

Although the WR blue bump was reported in the integrated spectra of HS 0837+4717 (Pustilnik et al. 2004) and Mrk 930 (Guseva et al. 2000), we detected it neither in any of the individual spaxels of our IFU observations nor in the integrated spectra of the brightest regions. The low $S / N$ of the bump combined with poor seeing conditions during the observing night could be the main reasons. Nonetheless, it is possible to carry on the spatial analysis of the physical properties and the $\mathrm{O}$ and $\mathrm{N}$ chemical abundances along the field of view of the IFU. The analysis described in Sect. 3.6 shows that, in most of the regions of the three galaxies, we can fit a Gaussian function to the $\mathrm{O}$ abundance and $\mathrm{N} / \mathrm{O}$ distributions, obtained from both the direct method and the strong-line calibration from $\mathrm{R} 23-\mathrm{P}$ for $\mathrm{O}$ abundance and $\mathrm{N} 2 \mathrm{O} 2$ for N/O. Besides this, the mean values of these Gaussian functions match the values derived using the integrated spectra from both the brightest region within the errors and the reported longslit observations in each galaxy. These values are consistent with a scenario of low metallicity with an excess of the N/O in relation to the expected value at this metallicity regime. Adopting $\log (\mathrm{N} / \mathrm{O})=-1.6$ as the most typical value of the plateau in the $12+\log / \mathrm{O} / \mathrm{H})$ vs. $\log (\mathrm{N} / \mathrm{O})$ relation, as can be seen in Fig. 1, this excess is $\Delta \log (\mathrm{N} / \mathrm{O})=+0.43 \mathrm{dex}$ in $\mathrm{HS} 0128+2832,+0.78 \mathrm{dex}$ in HS $0837+4717$ and +0.17 dex in Mrk 930, taking as a reference the mean value of the Gaussians fitted to the distributions of $\log (\mathrm{N} / \mathrm{O})$ as derived using the direct method in the respective Region 3 of the galaxies. At the same time, we can take the mean radii of the spatial distributions of $\log (\mathrm{N} / \mathrm{O})$ into account, considering the spatial size of the spaxels at the adopted distances for each galaxy, which are $330 \mathrm{pc}$ in HS $0128+2832,840 \mathrm{pc}$ in HS 0837+4717, and $380 \mathrm{pc}$ in Mrk 930. By inspecting the corresponding maps, the sizes of the $\log (\mathrm{N} / \mathrm{O})$ distributions as derived from the direct method are approximately $1.2 \times 1.7 \mathrm{kpc}^{2}$ for HS $0128+2832,2.5 \times 3.4 \mathrm{kpc}^{2}$ for HS $0837+4717$, and $1.9 \times 3.0 \mathrm{kpc}^{2}$ for Mrk 930, assuming that these are upper limits due to the seeing of the observing night.

These scale lengths lead us to question of whether the reported WR stars can be the actual source of the global N pollution detected in our sample of BCDs. To check the validity of this possibility we took the chemical yields of different elemental species ejected to the ISM by massive stellar clusters proposed by Mollá \& Terlevich (in prep.) for a metallicity of the cluster $Z=0.004\left(\approx 1 / 5 Z_{\odot}\right)$, which is close to the metallicity of our three observed BCDs. We studied the effects of these winds on a spherical homogeneous distribution of gas with constant density of $100 \mathrm{~cm}^{-3}$ and an initial $\mathrm{O}$ abundance of $12+\log (\mathrm{O} / \mathrm{H})=8.0$ and $\log (\mathrm{N} / \mathrm{O})=-1.6$. We also assumed that the mixing of the ejected elements with the material of the spherical distribution is instantaneous, which is not realistic at these galactic scales, but it is the most favorable hypothesis for the assumption of the pollution of the ISM by stellar winds.

The evolution of the $\log (\mathrm{N} / \mathrm{O})$ ratio with time is shown in Fig. 14 under different assumptions. In the left panel, we show the effect of the ejecta from a massive cluster with $\log \left(M / M_{\odot}\right)=$ 7 to a spherical gaseous distribution of different radii. As we can see, the effect is negligible for the typical radii of the N/O distributions in our galaxies, which are of the order of $1 \mathrm{kpc}$. For lower radii, it begins to be apparent how the ejection of this material changes the relative chemical composition of the ISM. At first, the ejection of $\mathrm{O}$ by very massive stars makes the N/O decrease, and the later appearance of WR star winds makes the N/O increase again, reaching a maximum and an asymptotic value. The value of this maximum matches only the measured mean values of HS $0128+2832$ and Mrk 930 for radii much lower than those measured in the IFU observations. For HS 0837+4717, the measured N/O is even higher than the maximum value of $\log (\mathrm{N} / \mathrm{O})$ reached at any radius.

In the right hand panel of Fig. 14 we fixed the radius of the spherical gas distribution to $1 \mathrm{kpc}$, and we varied the stellar 

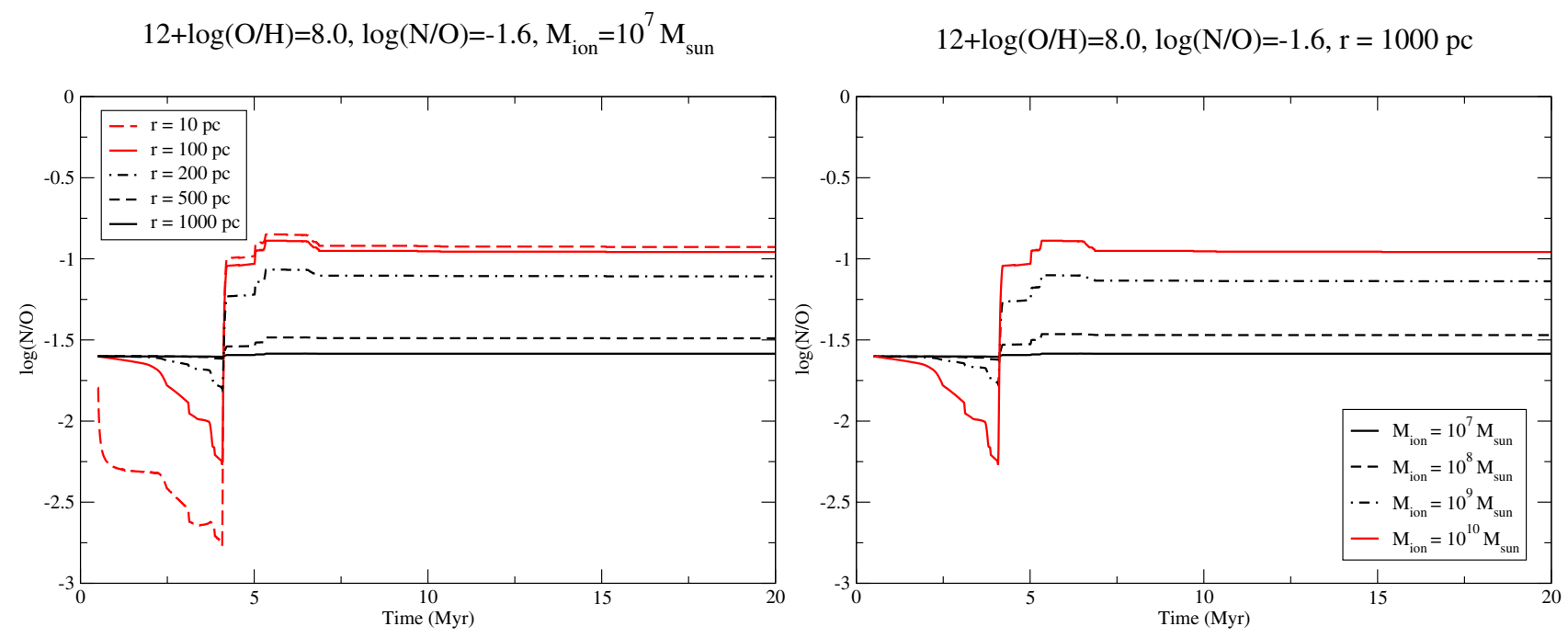

Fig. 14. Evolution of the N/O for a spherical gaseous distribution of constant density with an initial metallicity of $12+\log (\mathrm{O} / \mathrm{H})=8.0$ and $\log (\mathrm{N} / \mathrm{O})=-1.6$, which is polluted by the stellar winds coming from a stellar cluster with $Z=0.004$. To the left, we show the effects of a cluster of mass $\log \left(M / M_{\odot}\right)=7$ for different radii of the distribution. To the right, we show the effects of clusters of different stellar masses on a gaseous distribution with a radius of $1 \mathrm{kpc}$. Stellar wind yields were taken from Mollá \& Terlevich (in prep.).

mass of the ionizing cluster. As can be seen, the effects are only noticeable on this scale when the cluster is more massive than $\log \left(M / M_{\odot}\right)=8$. We can estimate the masses of the ionizing stellar clusters in the three BCDs, taking the following expression proposed by Díaz (1998)

$\log \left(M / M_{\odot}\right)=\log L(\mathrm{H} \alpha)-0.86 E W(\mathrm{H} \beta)-32.61$

with the $\mathrm{H} \alpha$ luminosities measured from the area covered by the IFU and the $E W(\mathrm{H} \beta)$ of the integrated spectrum of the corresponding brightest regions. Therefore, the ionizing masses are $10^{6.47} M_{\odot}$ for HS $0128+2832,10^{7.45} M_{\odot}$ for HS $0837+4717$, and $10^{7.72} M_{\odot}$ for Mrk 930. Although these estimates are only lower limits, because escaping photons and dust absorption are not considered, they are much lower than the mass of the ionizing cluster required to pollute the ISM on the spatial scales measured in our IFU observations.

Therefore, although a number of WR stars were detected in two of these galaxies (i.e. on the order of $10^{3}$ both in HS $0837+4717$ by Pustilnik et al. 2004 and in Mrk 930 by Guseva et al. 2000), and according to stellar evolutionary models they are also expected to be present in HS $0128+2832$, the overall high N/O observed in these objects is not likely to be produced by the pollution from stellar wind ejecta coming from WR stars, even in the most favorable conditions of mixing and dillution and, on the contrary, could be more related to other global processes affecting the metal content of the whole galaxy, as already suggested for the green pea galaxies analyzed by Amorín et al. (2010). They propose a combination of outflows of enriched gas and inflows of metal-poor gas, capable of triggering the star formation processes in these objects, decreasing the total content of metals without noticeably changing the N/O (Köppen \& Hensler 2005).

\section{Conclusions}

By means of PMAS optical integral field spectroscopy, we studied the spatial distribution of ISM physical-chemical properties for a sample of three BCDs (HS 0128+2832, HS 0837+4717, Mrk 930) with low metallicity and an excess of $\mathrm{N} / \mathrm{O}$, as measured with previous integrated long-slit spectrophotometry.
The spectral range covering from 3700 to $6900 \AA$ and the spatial resolution of $1^{\prime \prime} \times 1^{\prime \prime}$ in a field of view of $16^{\prime \prime} \times 16^{\prime \prime}$ ensure the characterization of the physical properties and the chemical abundances across the objects, although the spatial resolution is somehow reduced due to the seeing during the observing night $\left(\approx 2^{\prime \prime}\right)$.

The $\mathrm{H} \alpha$ intensity maps show that both HS $0128+2832$ and HS $0837+4717$ have a compact aspect, with their luminosities dominated by a single central massive stellar cluster. Mrk 930, whose morphology was also compared with available UV HSTACS images, shows a more complex structure, with knots in the northern and southern parts of the galaxy. Besides, it shows a clear rotation curve along the north-south axis, which could be the consequence of an interaction system.

All properties derived from the emission-line intensities with enough $S / N$ were analyzed in different spaxels of the field of view. Their corresponding distribution in different regions were also compared to the properties of the objects as derived from the integrated spectrum collected in the brightest region of each galaxy. For instance, while all integrated spectra lie in the starforming region of the $[\mathrm{NII}] / \mathrm{H} \alpha$ vs. [OIII] $/ \mathrm{H} \beta$ diagram, a sample of the spectra in the outer regions of each galaxy extends towards the right in this diagram, even lying in the so-called composite and AGN regions. This is due to the high N/O of these spaxels, combined with the excitation structure of the gas, causing the $[\mathrm{NII}]$ emission lines to remain bright even at very large distances from the ionizing stellar source.

We assumed that a certain property of the ISM can be considered as uniform within the galaxy or a determined region if the null hypothesis of the Lilliefors test (i.e. the fitting of a Gaussian function to the corresponding distribution) cannot be rejected. We found, that in those cases where a Gaussian does not fit a given distribution, the disagreement between the mean value of the distribution and the value derived from the integrated spectrum is higher.

We found that extinction, as derived from the decrement of Balmer, is only uniform in HS $0837+4717$. Mrk 930 shows a substructure of higher extinction in the outer southern part of the galaxy, which breaks the uniformity. Excitation, as estimated using the $\left[\mathrm{O}_{\mathrm{II}}\right] /[\mathrm{O}$ III $]$ emission-line ratio is only uniform in the innermost regions of HS $0837+4717$ and Mrk 930, possibly 
because the lower excitation in the outer parts of these galaxies, as the distance to the corresponding ionizing sources increases. Electron density was only estimated with enough confidence in Mrk 930, where a structure of high density appears in the southern part of the galaxy, so the distribution of this property cannot be considered as uniform.

Electron temperature was derived in a sample of spaxels of the three galaxies from the [OIII] $4363 \AA$ to [OIII] 4959, $5007 \AA$ emission line ratio. Its distribution appears as uniform in HS $0837+4717$ and Mrk 930. This is also the case for O chemical abundances and N/O as derived using these electron temperatures through the direct method. In the case of HS $0128+2832$, the N/O derived using the direct method is the only ISM property in this galaxy that is uniform after our analysis. The distribution of $\mathrm{O} / \mathrm{H}$ and N/O derived using strong-line methods are uniform in fewer regions, possibly due to the dependence of the corresponding strong-line parameters on the ionization structure of the gas.

Finally, we detected neither the broad WR blue bump ( $\sim 4650 \AA)$ nor the red one $(\sim 5808 \AA)$ suggested by previous works in two of the observed galaxies, even though we investigated whether these stars can be considered as the main cause of the measured excess of N/O. We used the stellar yields of massive star winds at the corresponding metallicity of these objects ( $Z=0.004$, Mollá \& Terlevich, in prep.) and taking the stellar masses of the ionizing clusters into account as estimated from the integrated $\mathrm{H} \alpha$ flux, to demonstrate that at the scale lengths where we detect the uniform N/O high values (i.e. $\sim 1 \mathrm{kpc}$ ), the WR stars cannot be responsible for the enhancement in the $\mathrm{N}$ abundance. It would be necesary between 2 and 3 orders of magnitude higher in the ionizing cluster mass to reach such a degree of pollution, assuming an instantaneous mixing of the ejected material with the surrounding gas. Thus, for the case of these three studied galaxies, another chemical evolution scenario is required (e.g. metal-rich outflows, infall of metal-poor gas, or another interacting scenario as the morphology and dynamics of Mrk 930 suggest).

Acknowledgements. Based on observations collected at the Centro Astronómico Hispano Alemán (CAHA) at Calar Alto, operated jointly by the Max-Planck Institut für Astronomie and the Instituto de Astrofísica de Andalucía (CSIC).

This work was partially supported by projects AYA2007-67965-C03-02 and AYA2007-67965-C03-03 of the Spanish National Plan for Astronomy and Astrophysics, by the project TIC114 Galaxias y Cosmología of the Junta de Andalucía and project CSD2006 00070 1st Science with GTC of the Spanish Ministry of Science and Innovation (MICINN).

Some of the data presented in this paper were obtained from the Multimission Archive at the Space Telescope Science Institute (MAST). STScI is operated by the Association of Universities for Research in Astronomy, Inc., under NASA contract NAS5-26555. Support for MAST for non-HST data is provided by the NASA Office of Space Science via grant NAG5-7584 and by other grants and contracts C.K., as a Humboldt Fellow, acknowledges support from the Alexander von Humboldt Foundation, Germany R.G.B. acknowledges additional funding by the China National Postdoc Fund Grant No. 20100480144

\section{References}

Adamo, A., Ostlin, G., Zackrisson, E., et al. 2011 [arXiv:1103.5771], MNRAS, in press

Alloin, D., Collin-Souffrin, S., Joly, M., \& Vigroux, J. M. 1979, A\&A, 78, 200 Amorín, R. O., Pérez-Montero, E., \& Vílchez, J. M. 2010, ApJ, 715, L128

Baldwin, J. A., Phillips, M. M., \& Terlevich, R. J. 1981, PASP, 93, 5 (BPT)

Brinchmann, J., Kunth, D., \& Durret, F. 2008, A\&A, 485, 657

Cardelli, J. A., Clayton, G. C., \& Mathis, J. S. 1989, ApJ, 345, 245

Chiappini, C., Hirschi, R., Meynet, G., et al. 2006, A\&A, 449, L27

Díaz, A. I. 1988, MNRAS, 231, 57

Díaz, A. I. 1998, Ap\&SS, 263, 143

Dray, L. M., Tout, C. A., Karakas, A. I., \& Lattanzio, J. C. 2003, MNRAS, 338 , 973

Edmunds, M. G., \& Pagel, B. E. J. 1978, MNRAS, 185, 77

Garnett, D. R. 1990, ApJ, 363, 142

Gavilán, M., Mollá, M., \& Buell, J. F. 2006, A\&A, 450, 509

Guseva, N. G., Izotov, Y. I., \& Thuan, T. X. 2000, ApJ, 531, 776

Hägele, G. F., Pérez-Montero, E., Díaz, Á. I., Terlevich, E., \& Terlevich, R. 2006, MNRAS, 372, 293

Hägele, G. F., Díaz, Á. I., Terlevich, E., et al. 2008, MNRAS, 383, 209

Henry, R. B. C., Nava, A., \& Prochaska, J. X. 2006, ApJ, 647, 984

Izotov, Y. I., \& Thuan, T. X. 1998, ApJ, 500, 188

Izotov, Y. I., \& Thuan, T. X. 2004, ApJ, 602, 200

James, B. L., Tsamis, Y. G., Barlow, M. J., et al. 2009, MNRAS, 398, 2

Kauffmann, G., Heckman, T. M., White, S. D. M., et al. 2003, MNRAS, 341, 33

Kehrig, C., Vílchez, J. M., Sánchez, S. F., et al. 2008, A\&A, 477, 813

Kewley, L. J., Dopita, M. A., Sutherland, R. S., Heisler, C. A., \& Trevena, J. 2001, ApJ, 556, 221

Kewley, L. J., Groves, B., Kauffmann, G., \& Heckman, T. 2006, MNRAS, 372, 961

Kobulnicky, H. A., \& Skillman, E. D. 1997, ApJ, 489, 636

Köppen, J., \& Hensler, G. 2005, A\&A, 434, 531

Kunth, D., \& Östlin, G. 2000, A\&ARv, 10, 1

Lilliefors, H. W. 1967, J. Amer. Statistical Assoc., 62, 399

López-Sánchez, Á. R., Esteban, C., García-Rojas, J., Peimbert, M., \& Rodríguez, M. 2007, ApJ, 656, 168

Lopez-Sanchez, A. R., Mesa-Delgado, A., Lopez-Martin, L., \& Esteban, C. 2011, MNRAS, 411, 2076

Marigo, P. 2001, A\&A, 370, 194

McCall, M. L., Rybski, P. M., \& Shields, G. A. 1985, ApJS, 57, 1

Meynet, G., \& Maeder, A. 2002, A\&A, 390, 561

Mollá, M., Vílchez, J. M., Gavilán, M., \& Díaz, A. I. 2006, MNRAS, 372, 1069

Pérez-Montero, E., \& Díaz, A. I. 2005, MNRAS, 361, 1063

Pérez-Montero, E., \& Contini, T. 2009, MNRAS, 398, 949

Monreal-Ibero, A., Vílchez, J. M., Walsh, J. R., \& Muñoz-Tuñón, C. 2010, A\&A, 517, A27

Pérez-Montero, E., \& Díaz, A. I. 2003, MNRAS, 346, 105

Pérez-Montero, E., García-Benito, R., Hägele, G. F., \& Díaz, Á. I. 2010 MNRAS, 404, 2037

Pilyugin, L. S. 1992, A\&A, 260, 58

Pilyugin, L. S., \& Thuan, T. X. 2005, ApJ, 631, 231

Prantzos, N. 2003, unpublished [arXiv:astro-ph/0301043]

Pustilnik, S., Kniazev, A., Pramskij, A., et al. 2004, A\&A, 419, 469

Renzini, A., \& Voli, M. 1981, A\&A, 94, 175

Roth, M. M., Kelz, A., Fechner, Th., et al. 2005, PASP, 117, 620

Sánchez, S. F. 2006, Astron. Nachr., 327, 850

Storey, P. J., \& Hummer, D. G. 1995, MNRAS, 272, 41

Terlevich, R., Melnick, J., Masegosa, J., Moles, M., \& Copetti, M. V. F. 1991, A\&AS, 91, 285

Thuan, T. X., Izotov, Y. I., \& Lipovetsky, V. 1996, ApJ, 463, 120

van den Hoek, L. B., \& Groenewegen, M. A. T. 1997, A\&AS, 123, 305

van Zee, L., Salzer, J. J., \& Haynes, M. P. 1998, ApJ, 497, L1

Ventura, P., D’Antona, F., \& Mazzitelli, I. 2002, A\&A, 393, 215

Vila-Costas, M. B., \& Edmunds, M. G. 1993, MNRAS, 280, 720

Walsh, J. R., \& Roy, J.-R. 1987, ApJ, 319, L57

Walsh, J. R., \& Roy, J.-R. 1989, MNRAS, 239, 297 